\title{
A Long-Stroke Planar Actuator with Multiple Degrees of Freedom by Minimum Number of Polyphase Currents
}

\author{
Yasuhito Ueda and Hiroyuki Ohsaki \\ The University of Tokyo \\ Japan
}

\section{Introduction}

There are a large number of drive systems employing numerous actuators in industry. As such, the performances of these actuators require constant improvement in terms of higher speed and precision, miniaturization, and lower energy consumption. In addition, most of these drive systems need a design that permits MDOF (Multi-Degree-Of-Freedom) motion. Motion controls allowing MDOF have been practically realized by using stacked multiple actuators (Ebihara et al., 2005). However, there are problems in attempting to improve the performance of these types of drive systems such as a larger and more complicated structure, fluctuation of the center of gravity, and Abbe errors in position measurement due to the multiple-moving parts. In order to eliminate these problems, MDOF actuators - which have only a single moving part, but are capable of being directly driven with MDOF - are emerging technologies for future applications (Ebihara et al., 2005).

Most MDOF actuators can be classified into two prominent types: a planar actuator that can drive in two-degree-of-freedom (2-DOF) translational directions; and, a spherical actuator that can drive in 2-DOF rotational directions. As for forms of the driving forces, electromagnetic, piezoelectric, magnetostrictive, and electrostatic types of MDOF actuators have been proposed (Ueda, 2009). Most models are of the electromagnetic actuator type because of mightiness and good controllability of the driving forces (Ueda, 2009).

This study deals with electromagnetic planar actuators, which have a mover capable of traveling over large translational displacements in a plane. The electromagnetic planar actuators that have been proposed can be classified by their drive principle such as stepping, direct-current, induction, and synchronous types. Synchronous planar actuators are expected to offer extremely good controllability of the motion controls, and furthermore in attempting to position a mover precisely, the mover should have no problematic wire in order to avoid heat generation at the mover and tension due to the wire that often deteriorates the drive characteristics. Therefore, synchronous planar actuators with a permanent-magnet mover have been studied actively. However, the movable area tends to be quite narrow due to the use of conventional magnetic circuits for the MDOF drives, which are spatially separated from one another as shown in Fig. 1 (Kim \& Trumper, 1998), unless the planar actuator has a large number of armature coils (Jeon et al., 2007). 


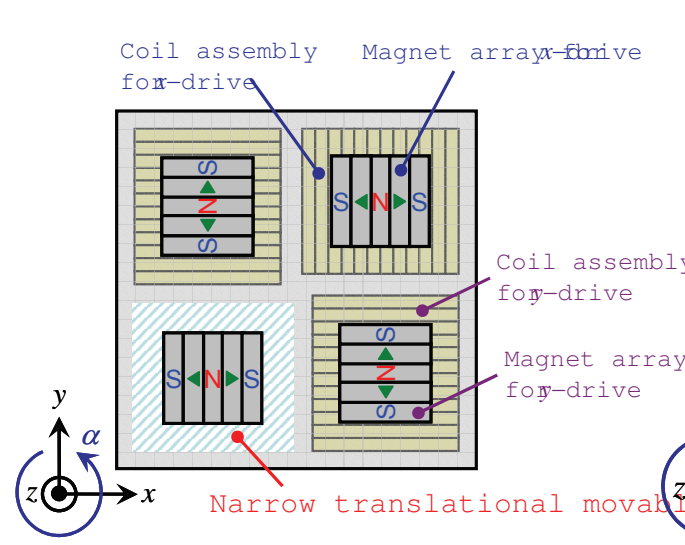

(a) When not displaced in $\alpha$.

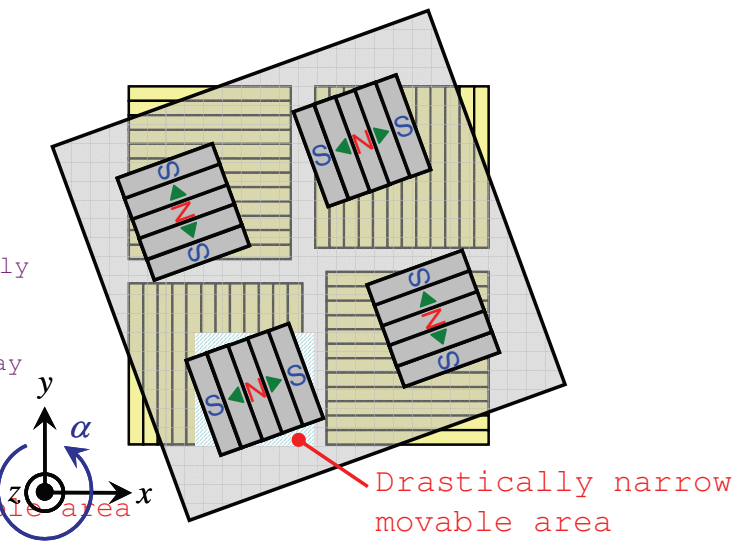

(b) When displaced in $\alpha$.

Fig. 1. Movable area of a prior planar actuator with spatially separated magnetic circuits

With this in mind, this study is aimed at designing high-performance planar actuators that have the following drive performances:

- decoupled control for 3-DOF (Three-Degree-Off-Freedom) motions on a plane.

- $\quad$ wide movable area that can be extended regardless of the number of armature coils.

- $\quad$ ease of mover miniaturization.

- no problematic wiring that can negatively influence drive performance.

- small number of armature currents to control.

Next, we propose a design for a novel synchronous planar actuator having spatially superimposed magnetic circuits for the 3-DOF drives. The magnetic circuits are a combination of a two-dimensional (2-D) Halbach permanent-magnet mover, and mutually overlapped stationary polyphase armature conductors. The movable area can be easily extended by increasing the length of the armature conductors, regardless of their number. However, independently controlling MDOF driving forces by means of superimposed magnetic circuits is very difficult and an extremely important issue in this study. This paper demonstrates a design for a planar actuator that enables MDOF driving forces to be controlled by using spatially superimposed magnetic circuits resulting from minimum number of armature currents.

First, based on the results of a numerical analysis of the driving forces, we design a decoupled control law for the 3-DOF driving forces on a plane by using two polyphase armature currents (Ueda \& Ohsaki, 2008a; Ueda \& Ohsaki, 2009). We experimentally demonstrate that the 3-DOF motions of the mover can be independently controlled by using two polyphase armature currents, and, therefore the planar actuator has the widest movable area of all planar actuators that have only two polyphase armature conductors.

Second, in order to further improve drive characteristics, the planar actuator is theoretically redesigned so that the mover can be stably levitated and the 3-DOF motions above a plane can be controlled by using three polyphase currents (Ueda \& Ohsaki, 2008b).

\section{3-DOF long-stroke planar actuator}

The fundamental structure of the 3-DOF planar actuator dealt with in this study is shown in Fig. 2. The planar actuator has a mover consisting of a 2-D Halbach permanent-magnet array 
and six stationary overlapped armature conductors, which are arranged in two layers of three.

\subsection{Fundamental structure}

\subsubsection{2-D Halbach permanent-magnet mover}

A Halbach permanent-magnet array generates a high-intensity magnetic field with a quasisinusoidal distribution along the array direction on one side of the magnet array (Halbach, 1986). Therefore, applying Halbach permanent-magnet arrays to coreless electric machines both enhances their driving forces and diminishes their force ripples (Zhu \& Howe, 2001). The mover, with which this study deals, consists of five-pole-and-nine-segment Halbach permanent-magnet arrays arranged two-dimensionally in the $x_{l}-$ and $y_{l}$-directions as shown in Fig. 3. Each permanent-magnet component was designed so as to maximize the ratio of the driving force of the planar actuator to the total mass of the mover at a nominal gap between the mover and stator, which is $0.5 \mathrm{~mm}$ (Ohsaki et al., 2003). The flux density $B_{z}$ is distributed quasi-sinusoidally in the $x_{l}$ - and $y_{l}$-directions, and can be approximately expressed near the mover center as follows:

$$
\left.B_{z}\left(x_{l}, y_{l} z\right)=B_{z m}(z) \quad c \oint_{\stackrel{s}{s}}^{\pi} x_{l}\right) \quad\left(\begin{array}{l}
\pi \\
\tau_{P M}^{\Theta s} \\
\tau_{P M}
\end{array} y_{l}\right)
$$

where $B_{z m}$ is a maximum flux density on a plane distant from the mover surface in the $z$-direction, and $\tau_{P M}$ is pole-pitch length in the $x_{l}$ and $y_{l}$-directions. Equation (1) can be rewritten by utilizing the $x_{m}-y_{m}$ coordinate, fixed with the mover, as follows:

$$
\left.B_{z}\left(x_{m}, y_{m} z_{2}\right)=\frac{B_{z m}(z)}{2}\left(\operatorname{c\phi } \underset{\tau}{\pi} x_{m}\right)+\left(\begin{array}{c}
\pi \\
\tau \\
\tau
\end{array} y_{m}\right)\right) .
$$

Equation (2) indicates that the permanent-magnet mover generates multipole magnetic fields superimposed spatially with the same lengths of pole pitch $\tau$ in the $x_{m}-$ and $y_{m}$-directions. Pole pitch $\tau$ in the $x_{m}$ - and $y_{m}$-directions can be expressed by the pole pitch $\tau_{P M}$ in the $x_{l}$ - and $y_{l}$-directions as follows:

$$
\tau=\tau_{P M} / \sqrt{2}
$$

\subsubsection{Stationary overlapped armature conductors}

There are two pairs of three stationary armature conductors arranged on two layers on a double-layered printed circuit board, for the $x$ - and $y$-directional drives. All the armature conductors are meander-shaped and are designed so that pitch length of the meander shape is $\tau$, corresponding to the pole-pitch length of the permanent-magnet mover in the $x_{m}$ - and $y_{m}$-directions. The three armature conductors arranged on each layer are mutually distant at $2 \tau / 3$ intervals, and supply the three-phase alternating currents for the $x$ - or $y$-directional drives. Between the two layers, there is a thin insulating layer that creates different gap lengths; the gap length between the mover and the armature conductors for the $x-$ directional drive is different from that between the mover and the armature conductors for the $y$-directional drive. So, it is extremely important to make the insulating layer as thin as possible. 


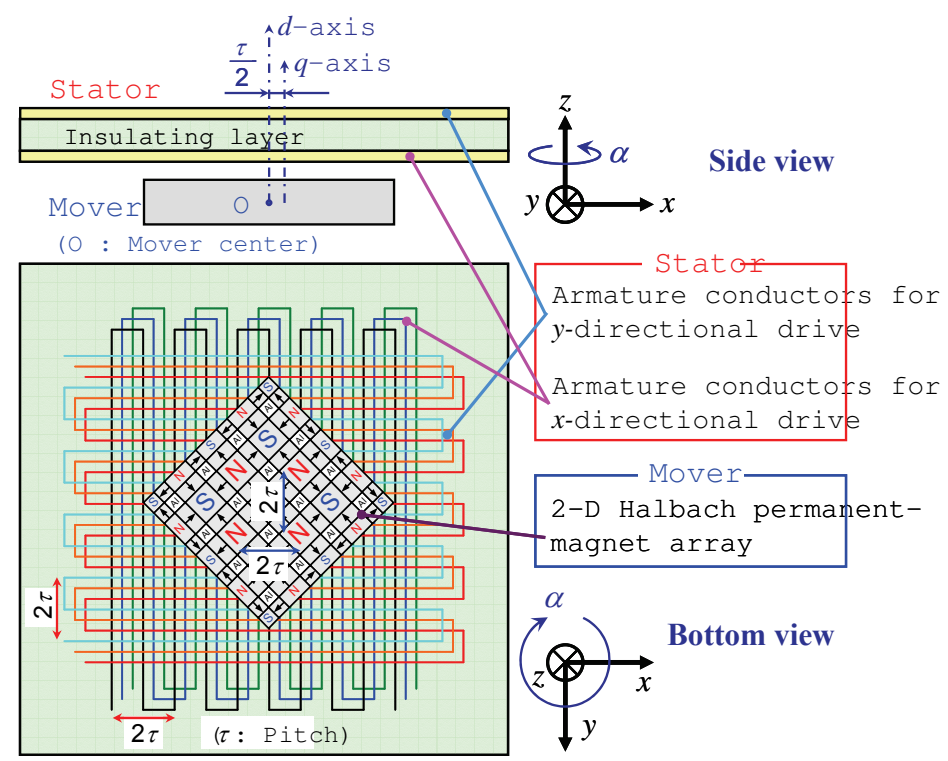

Fig. 2. Fundamental structure of planar actuator dealt with in this study

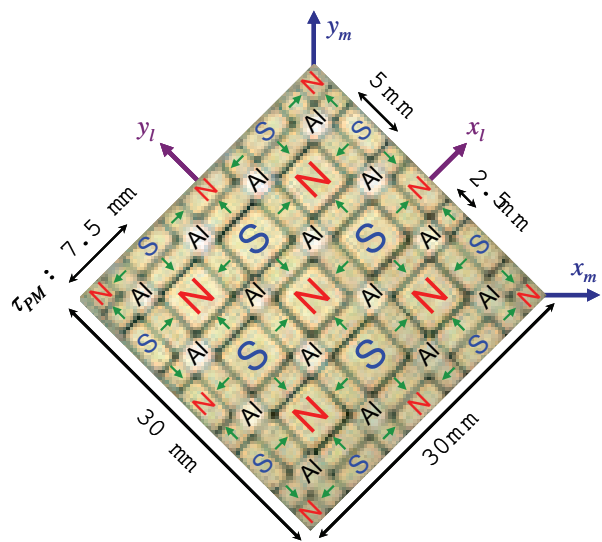

Fig. 3. Dimension and magnetization of permanent-magnet mover

All the armature conductors are arranged over the stator, and so are always subjected to the magnetic field for the $x$ - and $y$-directional drives generated by the permanent-magnet mover regardless of the mover position on the stator. Therefore, the mover can travel over the wide stator area. Furthermore, lengthening all the armature conductors extends the movable area without increasing the number of armature conductors, thus the powersupply system does not become complicated.

\subsubsection{Drive principle}

When the $y_{m}$ - and $x_{m}$-axes, fixed with the mover, are parallel to the armature conductors for the $x$ - and $y$-directional drives as shown in Fig. 2, the mover generates a quasi-sinusoidal 
flux-density distribution with pole pitch $\tau$, corresponding to the meander-shaped pitch in length, in the $x$ - and $y$-directions as shown in Eq. (2). Therefore, supplying two sources of three-phase alternating current to the two pairs of three armature conductors forms two magnetic circuits, as in linear synchronous motors, in the $x$ - and $y$-directions, and consequently generates driving forces in the $x$ - and $y$-directions. Although not all the magnetic circuits formed over the stator are mutually-separated, exciting the armature conductors for the $x$ - and $y$-directional drives independently generates driving forces in the $x$ - and $y$-directions, respectively, because of their mutually-orthogonal directions.

Displacing the $\alpha$-position (yaw position) and expressing the rotational position around the $z$-axis, not only influences the translational forces, it but also generates torque around the $z$-axis because of the broken symmetry of the spatial distribution of the translational forces acting on the mover. So it is extremely important to simultaneously control not only the $x-$ and $y$-motions, but also the $\alpha$-motions. Therefore, the characteristics of the translational forces and torque for the $\alpha$-positions need to be investigated in detail.

\subsection{Static force characteristics}

\subsubsection{Analytical model}

The driving forces acting on the mover of the planar actuator can be calculated from armature current $\boldsymbol{i}$ and flux density $\boldsymbol{B}$ using the Lorentz force equation $\boldsymbol{F}_{L}=\boldsymbol{i} \times \boldsymbol{B}$. Figure 4 shows the configuration of the mover and stator of the analysis model, and shows that the mover is displaced in the $\alpha$-direction and an armature current $i_{j k}$ is supplied to an armature conductor $l_{j k}$, where $j(=x$ or $y)$ and $k(=u, v$, or $w)$ express the driving direction and phase name of the three-phase currents, respectively. When a line element $d l_{j k}$, which is a small part of the armature conductor $l_{j k}$ is in the flux density $\boldsymbol{B}$, translational force $\boldsymbol{F}$ and torque $\boldsymbol{T}$ acting on the mover can be expressed as follows:

$$
\begin{gathered}
\boldsymbol{F}=-\sum_{j, k} \int_{l_{j k}}\left(\boldsymbol{i}_{j k} \times \boldsymbol{B}\right) d l_{j k} \\
\boldsymbol{T}=-\sum_{j, k} \int_{l_{j k}}\left(\boldsymbol{r}_{j k}-\boldsymbol{r}_{m}\right) \times\left(\boldsymbol{i}_{j k} \times \boldsymbol{B}\right) d l_{j k}
\end{gathered}
$$

where $\boldsymbol{r}_{j k}$ and $\boldsymbol{r}_{m}$ are position vectors of the line element $d l_{j k}$ and the mover center $\mathrm{O}^{\prime}$ with respect to the stationary coordinate $x_{s} y_{s} z_{s}$. From these equations, if the armature currents $\boldsymbol{i}_{j k}$ and flux density $\boldsymbol{B}$ are constant, the translational forces $\boldsymbol{F}$ and torques $\boldsymbol{T}$ are proportional to the square and cube of a side of the mover, respectively, because the lengths of the integration passes in Eqs. (4) and (5) are proportional to the square of a side of the mover. On the other hand, if mass density $\rho$ of the mover is constant, the mass and inertia-tensor elements of the mover are proportional to the third and fifth powers of a side of the mover, respectively. With this in mind, we can see that acceleration in the translational and rotational directions becomes twice and four times, respectively, when all sides of the mover become half.

In this analytical model, two pairs of three-phase currents $\left(i_{j u}, i_{j v}\right.$, and $\left.i_{j w}\right)$ are given as follows:

$$
i_{j u}=I_{j} \operatorname{sif}\left(\theta_{s j}\right)
$$




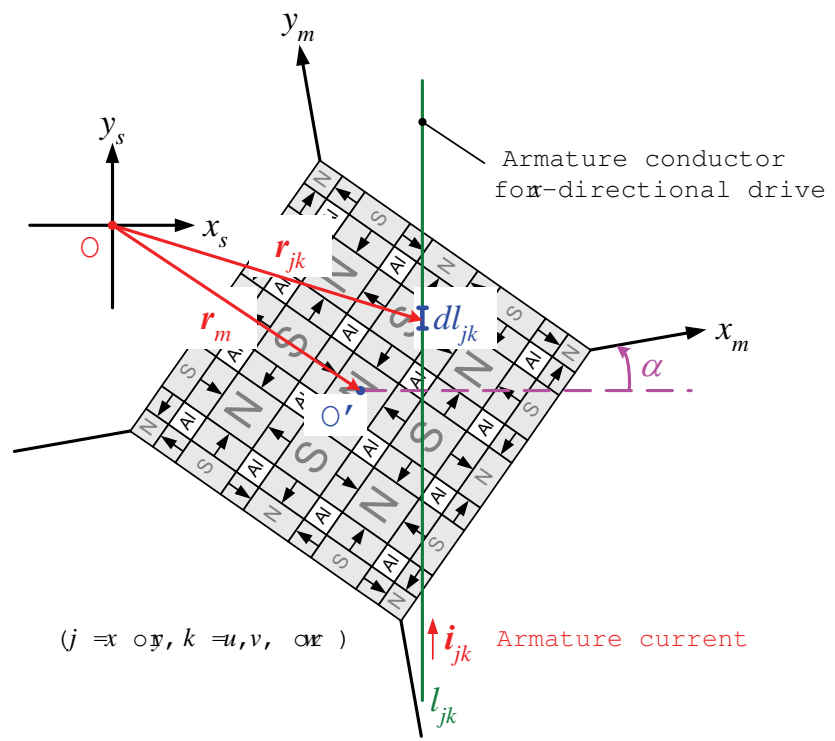

Fig. 4. Configuration of mover and stator in the analysis model

$$
\begin{aligned}
& \left.i_{j v}=I_{j} \operatorname{sif} \theta_{s j}+\frac{2 \pi}{3}\right) \\
& \left.i_{j w}=I_{j} \operatorname{sif} \theta_{s j}+\frac{4 \pi}{3}\right)
\end{aligned}
$$

where $I_{j}$ and $\theta_{s j}$ are the amplitude and the phase of the three-phase currents, respectively. A phase difference between magnetic fields generated by the armature conductors and the mover can be defined and expressed with the following equation:

$$
\theta_{d j}=\theta_{s j}-\frac{\pi}{\tau} j_{s}
$$

where $x_{s}$ and $y_{s}$ are the $x$ - and $y$-positions of the mover center $\mathrm{O}^{\prime}$ with respect to the stationary coordinate $x_{s} y_{s} z_{s}$. Controlling the amplitude $I_{j}$ and phase $\theta_{s j}$ of the armature conductors controls the 2-D mover motions.

\subsubsection{Numerical analysis results}

Exciting the armature conductors for the $x$ - and $y$-directional drives generates the $x$ - and $y$-directional driving forces using the same principle, and so this analysis deals with the armature conductors for only the $x$-directional drive.

When the $x_{m}$ - and $y_{m}$-axes are parallel to the stationary $x_{s}-$ and $y_{s}-$ axes, respectively, the yaw angle $\alpha$ is defined to be 0 deg. Figure 5 shows a numerical analysis result of the translational force $F_{x}$ and torque $T_{z}$ for the phase difference $\theta_{d x}$ with the following analysis conditions: 
- $\quad$ amplitude of the armature currents: $I_{x}=2 \mathrm{~A}, I_{y}=0 \mathrm{~A}$

- $\quad$ phase of the armature currents: $\theta_{s x}=-180 \sim 180 \mathrm{deg}$

- mover position: $x_{s}=0 \mathrm{~mm}$

- yaw angle: $\alpha=10 \mathrm{deg}$

- flux density due to the magnet mover: measurement result for air gap $=0.5 \mathrm{~mm}$.

Figure 5 indicates that the translational force $F_{x}$ and torque $T_{z}$ can be expressed as sinusoidal functions with respect to the phase difference $\theta_{d x}$. Equations (4) and (5) also indicate the translational force $F_{x}$ and torque $T_{z}$ are proportional to the amplitude of the armature currents, and therefore can be expressed as follows:

$$
\begin{aligned}
& F_{x}=K_{F x}(\alpha) I_{x} \operatorname{si\theta } \theta_{d x} \\
& T_{z}=K_{T x}(\alpha) I_{x} \cos \theta_{d x} .
\end{aligned}
$$

These equations indicate that phases of the armature currents generating the translational force $F_{x}$ and torque $T_{z}$ differ by $90 \mathrm{deg}$. Furthermore, the translational forces $F_{x}$ are proportional to the armature currents generating a 90-deg phase-lead magnetic field with respect to the magnetic field due to the magnet mover $\left(\theta_{d x}=90 \mathrm{deg}\right)$, and the torques $T_{z}$ are proportional to the armature currents generating the same-phase magnetic field $\left(\theta_{d x}=0\right.$ deg).

The system constants $K_{F x}$ and $K_{T x}$ depend on the yaw angle $\alpha$. The system constants $K_{F}$ and $K_{T}$ can be calculated by fitting the analysis results of the driving forces to Eqs. (10) and (11). Figure 6 shows the calculation results of the system constants $K_{F x}$ and $K_{T x}$. The same driving forces can be generated every $90 \mathrm{deg}$ in the $\alpha$-direction because of the symmetric structure of the permanent-magnet mover. Figure 6 shows a period of the system constants $K_{F x}$ and $K_{T x}$ in the $\alpha$-direction. Figure 6 indicates that $K_{F x}$ is maximum and $K_{T x}=0 \mathrm{~N} \cdot \mathrm{m} / \mathrm{A}$ when the yaw angle $\alpha=0 \mathrm{deg}$, and so the translational force $F_{x}$ is maximum and the torque $T_{z}$ is not generated. When the mover is displaced in the $\alpha$-direction, $K_{F x}$ becomes smaller and $K_{T x} \neq 0$ $\mathrm{N} \cdot \mathrm{m} / \mathrm{A}$. The driving forces can be generated in the range within the yaw angle $\alpha= \pm 30 \mathrm{deg}$ because of $K_{F x} \neq 0 \mathrm{~N} / \mathrm{A}$ and $K_{T x} \neq 0 \mathrm{~N} \cdot \mathrm{m} / \mathrm{A}$. The movable area in the $\alpha$-direction is widest of all planar actuator having only two pairs of polyphase conductors. Therefore, this planar actuator is suitable for 3-DOF long-stroke planar manipulation using only a few conductors.

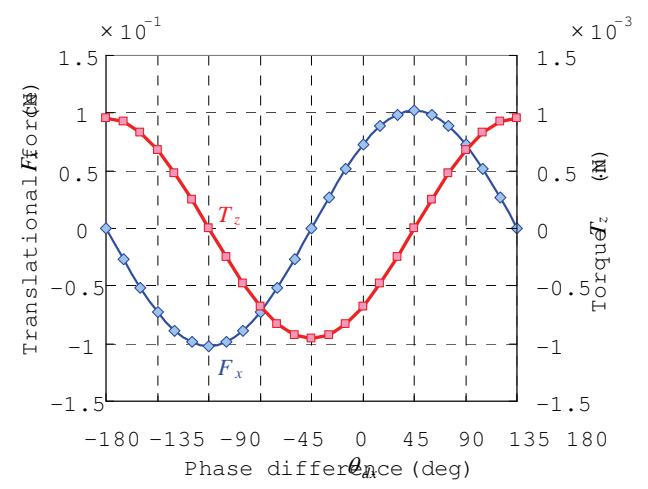

Fig. 5. Translational force $F_{x}$ and torque $T_{z}$ vs. phase difference $\theta_{d x}$ 


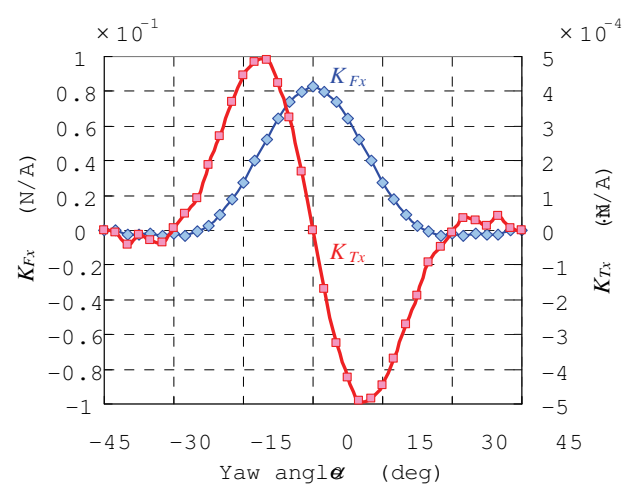

Fig. 6. System constants $K_{F x}$ and $K_{T x}$ vs. yaw angle $\alpha$

\subsection{Decoupled control for 3-DOF motions}

\subsubsection{DQ decomposition}

DQ decomposition in conventional rotary machines separates armature-current components that are generating torques from those that are not (Fitzgerald et al., 1990). From preciously shown analysis results, DQ decomposition in this planar actuator separates the armaturecurrent components, generating the translational forces $F_{x}, F_{y}$ or torques $T_{z}$.

The direct axis ( $d$-axis) and quadrature axis ( $q$-axis) are attached to the mover, and move together with the mover. In a phasor diagram, conventionally, the $d$-axis is aligned with the magnetic field axis because of the permanent-magnet mover, and the $q$-axis leads the $d$-axis by $90 \mathrm{deg}$. In other words, the $d$-axis current is intended to generate the same-phase magnetic field as that resulting from the permanent-magnet mover, and the $q$-axis current is intended to generate a 90-deg phase-lead magnetic field. Figure 7 shows phasor diagrams for the relation between the $d q$-frame and $u v w$-frame. The $u-, v-$, and $w$-phase currents generate magnetic fields with definite phases, which are out of phase from one another by $120 \mathrm{deg}$. The $\alpha^{\prime}$-axis current generates the same-phase magnetic field as that from the

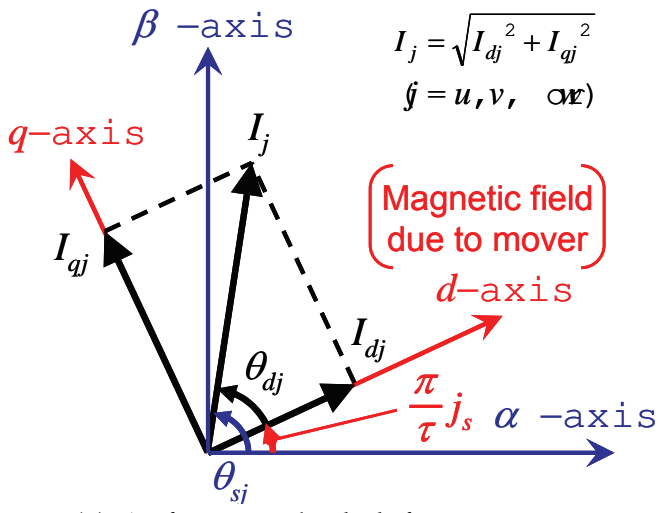

(a) $d q$-frame and $\alpha^{\prime} \beta^{\prime}$-frame.

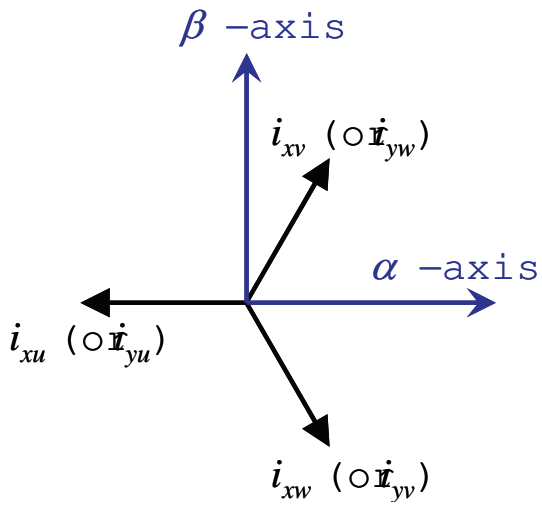

(b) $\alpha^{\prime} \beta^{\prime}$-frame and $u v w$-frame.

Fig. 7. Phasor diagrams showing relation between $d q$-frame and $u v w$-frame 
permanent-magnet mover when the mover center is at the origin of the stationary coordinate $x_{s} y_{s} z_{s}$. The $\beta^{\prime}$-axis leads the $\alpha^{\prime}$-axis by $90 \mathrm{deg}$. Figure 7 indicates that the $d$ - and $q$-axes lead the $\alpha^{\prime}$ - and $\beta^{\prime}$-axes by $\left(\pi j_{s} / \tau\right)$, respectively, which is proportional to the mover positions $j_{s}\left(=x_{s}\right.$ or $\left.y_{s}\right)$. The armature currents having the amplitude $I_{j}$ and phase $\theta_{s j}$ can be decomposed to the $d$-axis currents $I_{d j}$ and $q$-axis currents $I_{q j}$ as follows:

$$
\begin{gathered}
I_{d j}=I_{j} \operatorname{si} \Theta_{d j} \\
I_{q j}=I_{j} \cos \theta_{d j} .
\end{gathered}
$$

From Eqs. (10)-(13), the translational forces $F_{x}, F_{y}$ and torques $T_{z}$ resulting from supplying the two pairs of three-phase currents to the armature conductors for the $x$ - and $y$-directional drives, can be expressed with the $d$ - and $q$-axis currents $I_{d j}, I_{q j}$ as follows:

$$
\begin{gathered}
F_{x}=K_{F x}(\alpha) I_{q x} \\
F_{y}=K_{F y}(\alpha) I_{q y} \\
T_{z}=K_{T x}(\alpha) I_{d x}+K_{T y}(\alpha) I_{d y} .
\end{gathered}
$$

Equations (14)-(16) indicate that the translational forces $F_{x}, F_{y}$ and torques $T_{z}$ are proportional to the $q$ - and $d$-axis currents $I_{q j}, I_{d j}$, respectively. The system constants for the $y$-directional drive $K_{F y}, K_{T y}$ are slightly different from those for the $x$-directional drive $K_{F x}$, $K_{T x}$ because of difference in the air gaps between the mover and armature conductors for the $x$ - or $y$-directional drives. As mentioned above, the driving forces from the armature currents can be simply described.

\subsubsection{3-DOF force control}

Supplying the appropriate $d$ - and $q$-axis currents independently controls the translational forces $F_{x}, F_{y}$ and torques $T_{z}$ from Eqs. (14)-(16). The degrees of freedom for the armaturecurrent controls are four $\left(I_{d x}, I_{d y}, I_{q x}\right.$, and $\left.I_{q y}\right)$, and one larger than that for the mover motions, which is three $(x, y$, and $\alpha)$ as shown in Fig. 8. Controlling the two $q$-axis currents $I_{q x}, I_{q y}$ is essential to controlling the two translational forces $F_{x}, F_{y}$. The two $d$-axis currents $I_{d x}, I_{d y}$ generate the torques $T_{z}$, and, so the torque controls have redundancy. In fact, the $d$-axis current $I_{d x}$ generates the torques $T_{z}$ more efficiently than the $d$-axis current $I_{d y}$ because of the air-gap difference $\left(K_{T x}>K_{T y}\right)$. However, each armature current is limited by its own rating, power supply, and so on. In Fig. $8, I_{c}$ is the current limit of all the armature currents. So if large driving forces $F_{x}, F_{y}, T_{z}$ are required, then armature currents should be supplied so as to optimally satisfy the references of the driving forces under the current limit for producing maximum performance. This study, however, focuses principally on the verification of decoupled 3-DOF motion controls for a long-stroke planar actuator, and, therefore deals with a simply decoupled control algorithm.

Figure 9 shows a control block diagram for the translational forces $F_{x}, F_{y}$ and torques $T_{z}$ in this study. In Fig. 9, $x_{\text {ref }}, y_{r e f}$, and $\alpha_{r e f}$ are references of the mover positions in the $x-, y-$, and $\alpha$-directions, respectively. In order to decide the references for the driving forces $F_{x}{ }^{*}, F_{y}{ }^{*}, T_{z}{ }^{*}$, 
feedback controls in each degree of freedom are performed with three different PID (Proportional-Integral-Derivative) algorithms. The PID parameters are determined so that settling time of the $x-, y_{-}$, and $\alpha$-directional drives is shorter than $0.5 \mathrm{~s}$. In this study, references of the $d$ - and $q$-axis currents $I_{d j}{ }^{*}, I_{q j}{ }^{*}$ are calculated from the system constants $K_{F j}$, $K_{T j}$ and the driving force references $F_{x}{ }^{*}, F_{y}{ }^{*}, T_{z}{ }^{*}$ as follows $(j=x$ or $y)$;

$$
\begin{gathered}
I_{d j}{ }^{*}=T_{z}^{*} / K_{T j}(\alpha) \\
I_{q j}{ }^{*}=F_{j}^{*} / K_{F j}(\alpha) .
\end{gathered}
$$

The system constants $K_{F j}, K_{T j}$ are calculated from the detected yaw angle $\alpha$ by interpolation of the analysis data shown in Fig. 6. As we can see from Fig. 7 (a), the references of the amplitude and phase of the three-phase currents $I_{j}^{*}$ and $\theta_{s j}{ }^{*}$ can be calculated from the current references in the $d q$-frame $I_{d j}{ }^{*}, I_{q j}{ }^{*}$ and the mover positions in the $x$-and $y$-directions. Then, from Fig. 7 , references of the three-phase currents $i_{j u}{ }^{*}, i_{j v}{ }^{*}, i_{j w}{ }^{*},(j=x$ or $y)$ can be calculated from the amplitude $I_{j}^{*}$ and phase $\theta_{s j}{ }^{*}$.

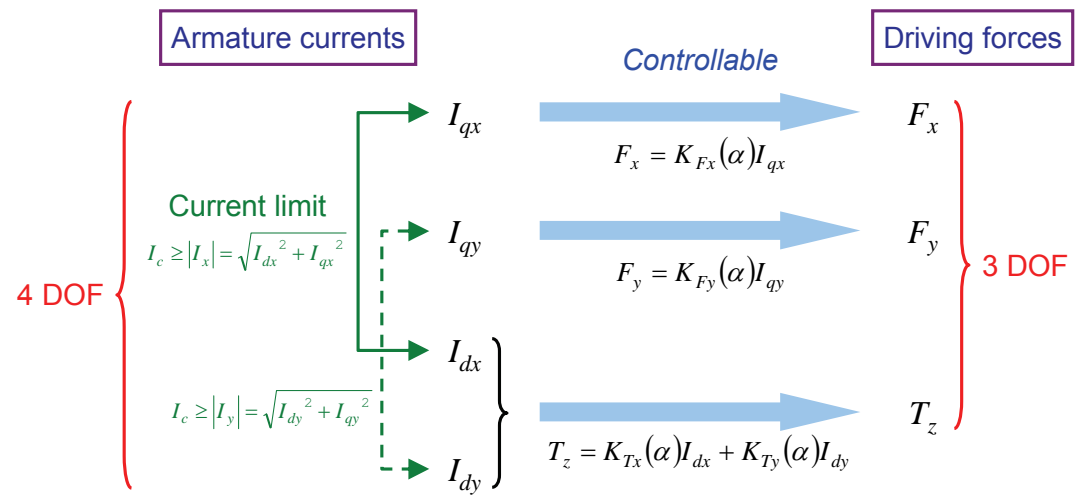

Fig. 8. Degrees of freedom for armature-current control and mover motion

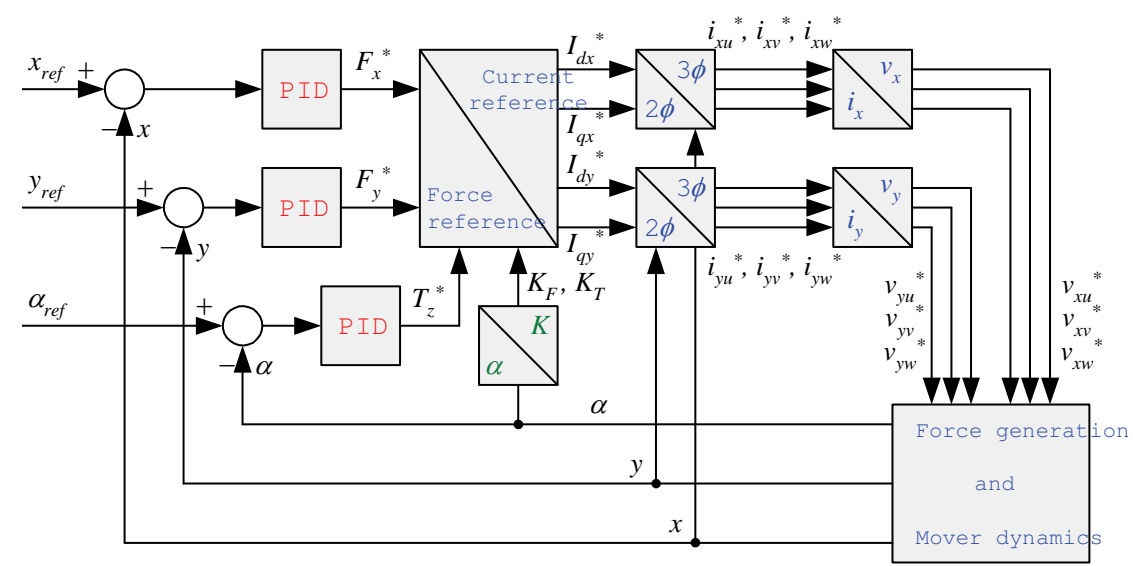

Fig. 9. Control block diagram for translational forces $F_{x}, F_{y}$ and torques $T_{z}$ 
In this study, each-phase armature voltage $v_{j k}{ }^{*}$ is calculated from the resistances $R$ and armature-current references $i_{j k}{ }^{*}$ as follows;

$$
v_{j k}{ }^{*}=R i_{j k}{ }^{*} .
$$

Equation (19) does not consider back electromotive force, and causes errors between the armature currents and their references. In this study, the errors are compensated by the PID controls. As described above, the six armature-voltage references $v_{j k}{ }^{*}$ can be calculated from the driving force references $F_{x}{ }^{*}, F_{y}{ }^{*}, T_{z}{ }^{*}$. Supplying the six armature voltages $v_{j k}{ }^{*}$ generates decoupled 3-DOF driving forces.

\subsubsection{Experimental setup}

In order to control the 3-DOF $(x, y$, and $\alpha)$ motions of the mover by position feedback, sensing the mover positions which are allowed anywhere on the wide stator area is extremely important. If multiple sensors are utilized for single-axis displacements, we require three or more sensors to detect the 3-DOF positions. The combination method for multiple sensors determines the measurement performance, for instance, detection accuracy and measurable area, and so is also extremely important. This study uses three single-axis laser-displacement sensors (laser triangulation) because of their long measurable area (several tens of $\mathrm{mm}$ ) and high resolution (several $\mu \mathrm{m}$ ).

When the mover is driven in 3-DOF directions, suspending and smoothly guiding the mover on a plane are also extremely important. This study uses ball bearings, 1-mm glass spheres, as the suspension and guide mechanism because they have smaller friction forces and are easily installed.

Figure 10 shows the configuration of the experimental system for the 3-DOF motion controls of the mover. The mover is supported by many ball bearings and guided on a plane having small frictional forces. At the same time, the mover faces the three-phase armature conductors for the $x$ - and $y$-directional drives through $0.5-\mathrm{mm}$ and $0.63-\mathrm{mm}$ air gaps, respectively. The three laser-displacement sensors irradiate three points on three lateral sides of the mover, and detect displacements at the three points by triangulation. A personal computer (PC), running a Digital Signal Processor (DSP), inputs the three sensor signals and calculates the 3-DOF positions of the mover. The PC also calculates the six armature-voltage references based on a decoupled motion-control algorithm, and outputs the six voltages to the six armature conductors through the power amplifiers. Then, the armature currents and magnetic field, resulting from the magnet mover, interact and independently controlled driving forces are generated.

\subsubsection{Experimental results}

This subsection presents verification of the 3-DOF drive characteristics of the planar actuator ascertained by experimental study, and describes the experimental results under various conditions. First, in order to verify the decoupled 3-DOF motion controls of the planar actuator with superimposed magnetic circuits, drive tests of a mover with 3 DOF were performed. Second, the movable area in the $\alpha$-direction was experimentally verified.

In this planar actuator, even if a large $\alpha$-displacement of the mover occurs, the driving forces decrease less than conventional planar actuators because the magnetic circuits for the $x-, y-$, and $\alpha$-directional drives are always formed. The planar actuator has a mover capable of infinitely-large translational motions on a plane by increasing the length of the armature conductors, in principle, as mentioned in Subsection 2.1.2. 


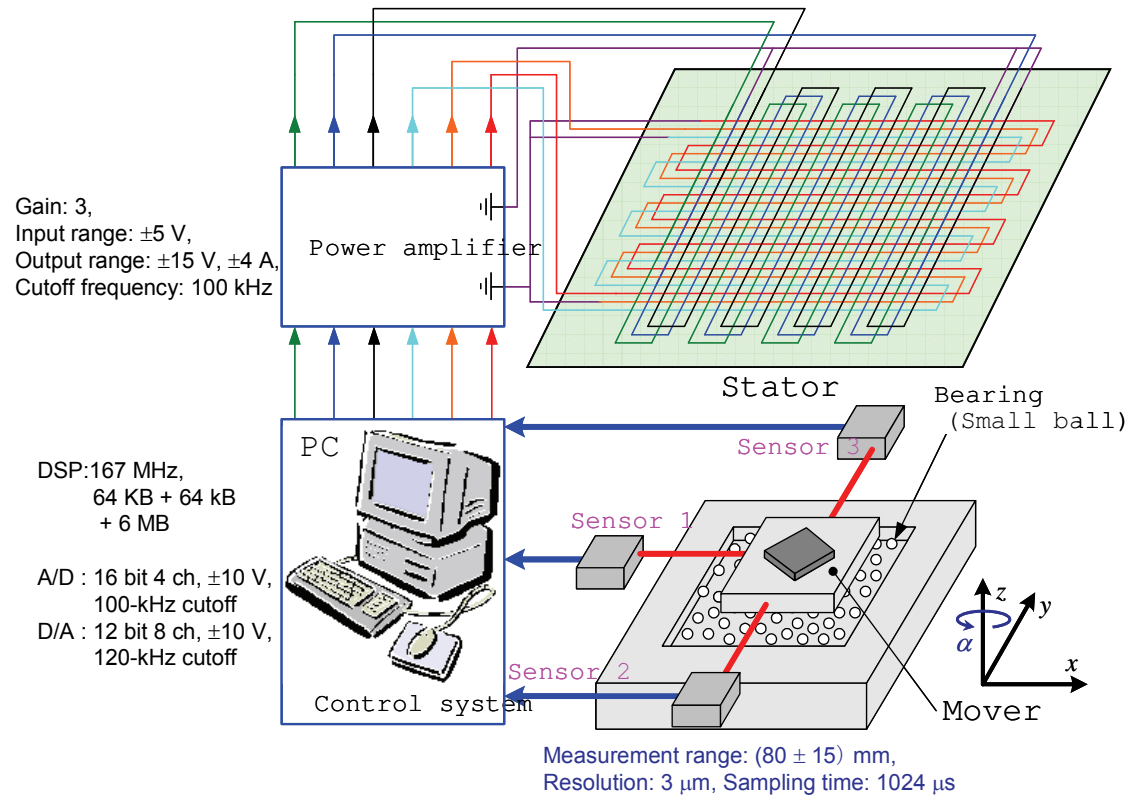

Fig. 10. Configuration of experimental system for 2-D drives

Next, in order to verify the long-stroke motion-control characteristics with 3 DOF, experimental results of the drive tests were described under the following four conditions:

(I) Simultaneous sine response for the $x-, y$ - and $\alpha$-directions (Ueda \& Ohsaki, 2008a); Verification of decoupled motion controls in the $x-, y$ - and $\alpha$-directions, and an evaluation of follow-up controls are performed.

Figure 11 shows the experimental results of the mover motions when sine signals, which have a 10-mm amplitude and a 2-s period, are simultaneously given as the $x-, y-$, and $\alpha$-position references. Figure 11 indicates that the mover can simultaneously track the three sine-reference positions over a wide movable area with less position error.

(II) $\quad \alpha$-directional drive at the yaw angle $\alpha \approx 0 \operatorname{deg}$ (Ueda \& Ohsaki, 2009):

Whereas the translational forces in the $x$ - and $y$-directions are maximum at the yaw angle $\alpha$ $=0 \mathrm{deg}$, torque in the $\alpha$-direction cannot be generated, as mentioned in Subsection 2.2.2. Therefore, controllability of the $\alpha$-motion is presumed to deteriorate at the yaw angle $\alpha \approx 0$ deg. Then, in order to verify the motion-control characteristics at the yaw angle $\alpha \approx 0 \mathrm{deg}$, ramp response for the $\alpha$-direction was investigated in the same range.

Figure 12 shows the experimental results of the mover motions and armature currents for ramp response with the position references $\left(x_{r e f}, y_{r e f}\right)=(0,0)$ and $\alpha_{\text {ref }}=-5+t(\mathrm{deg})$, where $t$ is time. Figure 12 indicates that the mover can travel in the $\alpha$-direction in the range within the yaw angle $\alpha= \pm 5 \mathrm{deg}$. When the yaw angle $\alpha$ closes to $0 \mathrm{deg}$, the large $d$-axis currents $I_{d x}$ and $I_{d y}$ are required to control the $\alpha$-position. In the range within the yaw angle $\alpha= \pm 1 \mathrm{deg}$, the $\alpha$-position becomes more oscillatory. The current capacity of the power amplifiers is presumed to be the reason the $d$-axis currents are insufficient to control the $\alpha$-motion, and consequently cause the oscillation in the $\alpha$-position. 


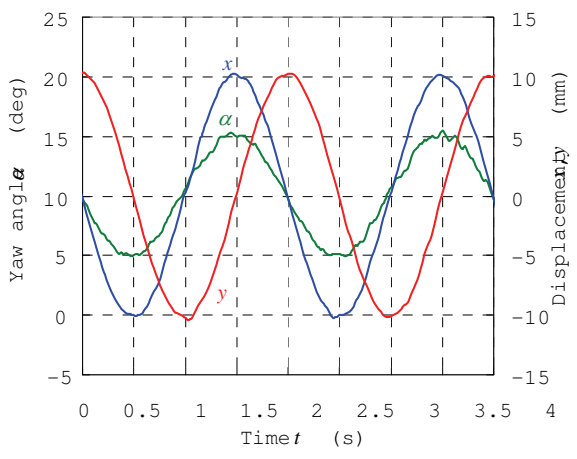

(a) Mover position.

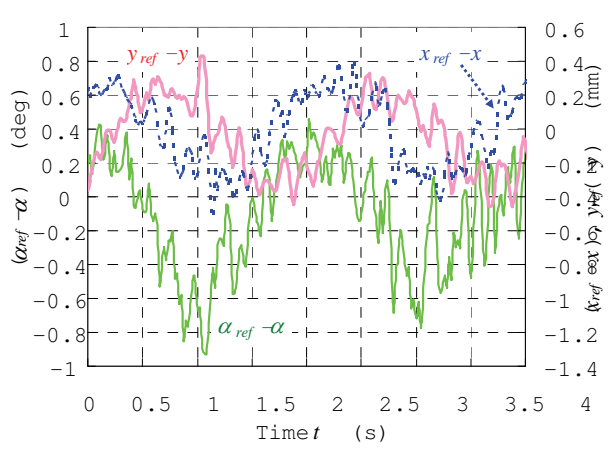

(b) Position error.

Fig. 11. Experimental results of simultaneous sine response for $x-, y-$ and $\alpha$-directions

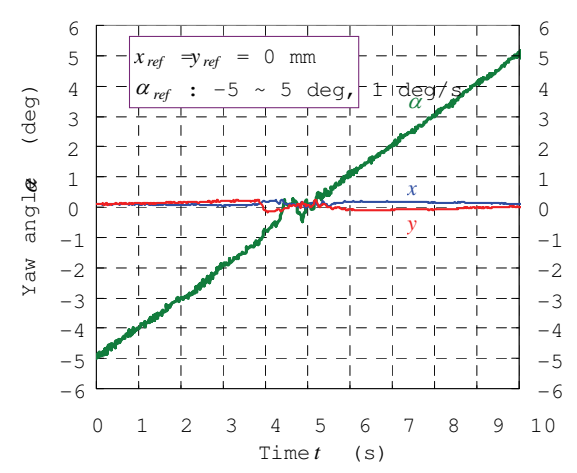

(a) Mover position.

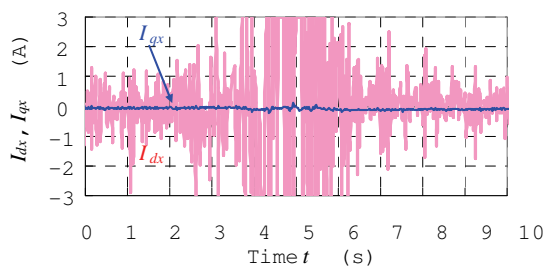

(c) Armature currents for the $x$-drive.

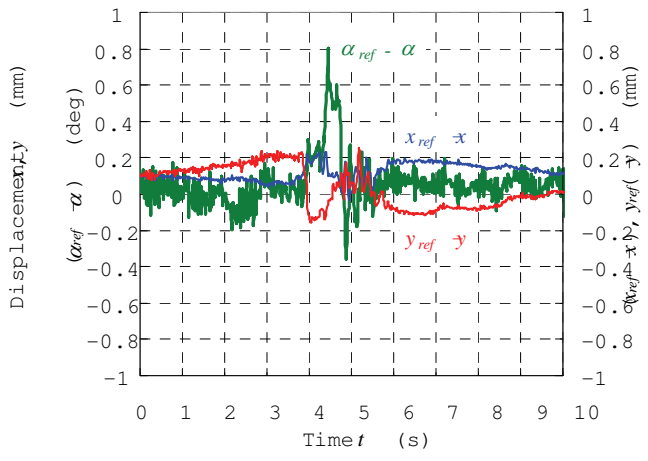

(b) Position error.

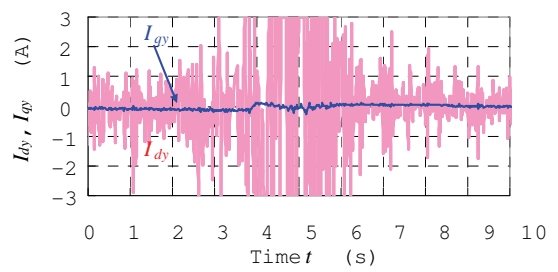

(d) Armature currents for the $y$-drive.

Fig. 12. Experimental results of ramp response for $\alpha$-direction at yaw angle $\alpha \approx 0$ deg

(III) $\quad \alpha$-directional drive at the yaw angle $\alpha>20 \mathrm{deg}$ (Ueda \& Ohsaki, 2009):

Increasing the yaw angle $\alpha$ can decrease the driving forces especially at the yaw angle $\alpha>20$ deg. Then, in order to verify the movable area and motion-control characteristics at the yaw angle $\alpha>20 \mathrm{deg}$, ramp response for the $\alpha$-direction was investigated in the same range.

Figure 13 shows the experimental results of the mover motions and armature currents for ramp response with the position references $\left(x_{r e f}, y_{r e f}\right)=(0,0)$ and $\alpha_{r e f}=18+t$ (deg). Figure 13 indicates that the mover can travel in the $\alpha$-direction at the yaw angle $\alpha<26 \mathrm{deg}$. When the yaw angle $\alpha$ becomes larger, the large $q$-axis currents $I_{q x}$ and $I_{q y}$ are required to control the 


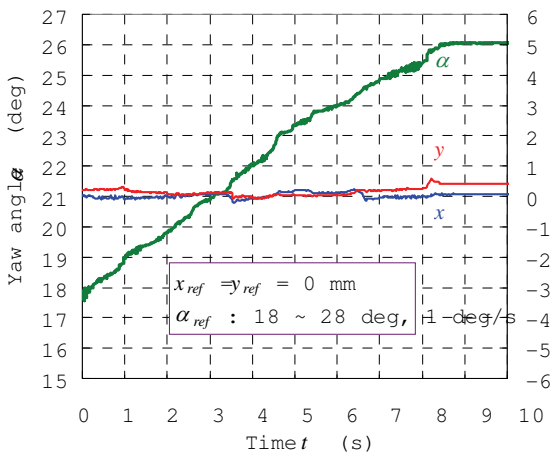

(a) Mover position.

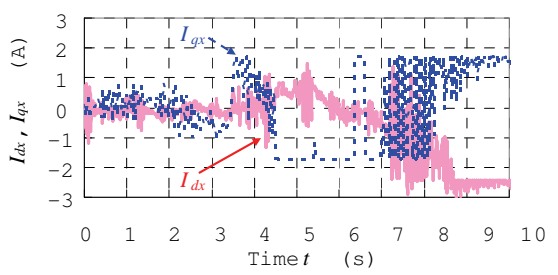

(c) Armature currents for the $x$-drive.

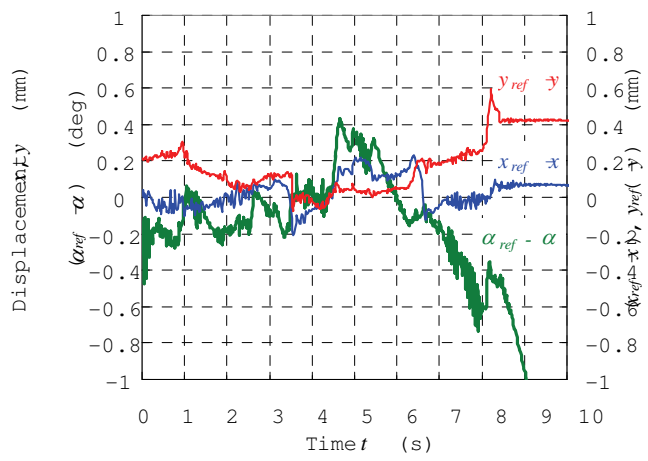

(b) Position error.

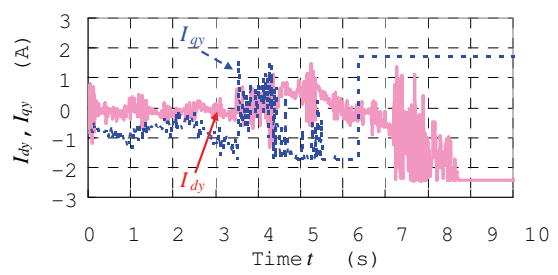

(d) Armature currents for the $y$-drive.

Fig. 13. Experimental results of ramp response for $\alpha$-direction at yaw angle $\alpha>20 \mathrm{deg}$ $x$ - and $y$-positions. At the yaw angle $\alpha>22 \mathrm{deg}$ (time $t>4 \mathrm{~s}$ ), the $q$-axis currents $I_{q x}$ and $I_{q y}$ are limited by the maximum current $=1.7 \mathrm{~A}$. At the yaw angle $\alpha>26 \mathrm{deg}$ (time $t>8 \mathrm{~s}$ ), the mover is stationary, and, therefore the driving forces required to overcome the friction forces between the mover and ball bearings cannot be generated.

(IV) $90-$ deg step response for the $\alpha$-direction (Ueda \& Ohsaki, 2009):

The driving forces of the planar actuator have a 90-deg periodicity for the $\alpha$-direction because of the symmetric magnetized mover. Furthermore, the driving forces are generated in the range within the yaw angle $\alpha= \pm 26 \mathrm{deg}$ in the 90-deg periodicity. Then, in order to verify feasibility of periodic 90-deg stepping drives in the $\alpha$-direction, a 90-deg step response was investigated.

Figure 14 shows experimental results of the mover motions and armature currents for 90deg step response with the initial positions $\left(x_{i n}, y_{i n}, \alpha_{i n}\right)=(0,0,10)$ and the position references $\left(x_{\text {ref }}, y_{\text {ref }}, \alpha_{\text {ref }}\right)=(0,0,100,190,280$, or 370). Figure 14 indicates that a 90 -deg stepping drive can be realized. The mover positions could not be detected in the range within $0.10 \mathrm{~s}<$ time $t<0.28 \mathrm{~s}$ due to geometrical problem in laser triangulation. At the yaw angle $\alpha>(14+90) \mathrm{deg}$, corresponding to time $t>0.28 \mathrm{~s}$, the mover motions can be controlled because of the $90-\mathrm{deg}$ periodicity in the yaw direction.

\subsection{Summary of chapter 2}

This chapter proposed a novel synchronous planar actuator having advantages in terms of the wide movable area of the magnet mover, which is independent of the number of armature conductors, presents the design for the experimental system for verification of the 


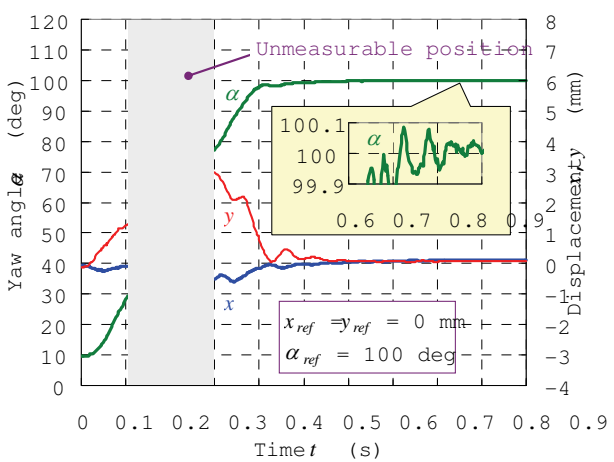

(a) Mover position $(0<t<1)$.

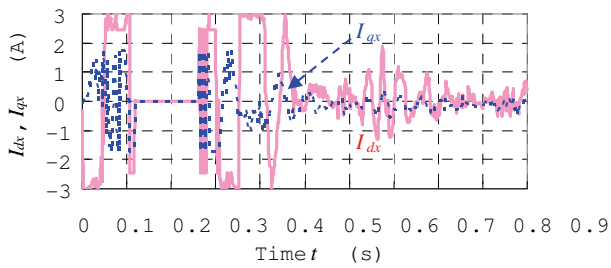

(c) Armature currents $(0<t<1)$.

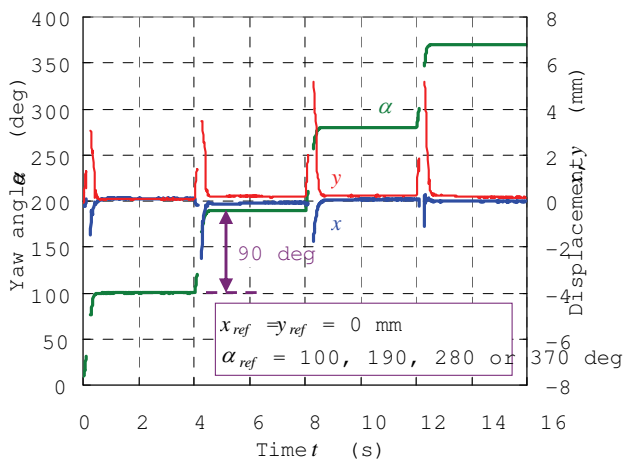

(b) Mover position $(0<t<16)$.

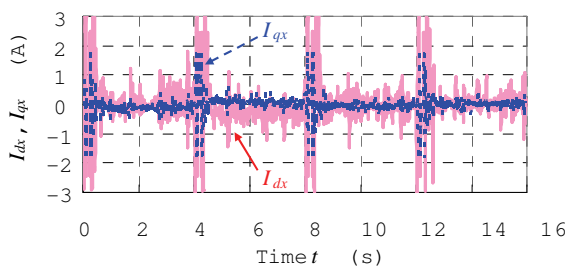

(d) Armature currents $(0<t<16)$.

Fig. 14. Experimental results of 90-deg stepping response for $\alpha$-direction

drive characteristics of this long-stroke planar actuator, and describes the experimental results of long-stroke 3-DOF motion controls. From these results, we successfully demonstrated that the 3-DOF motions of the mover can be independently controlled by two polyphase armature currents. The movable area in the $x$ - and $y$-directions is infinitely wide in principle, and that in the $\alpha$-direction is in the range within $\pm 26 \mathrm{deg}$, meaning the planar actuator has the widest movable area of all planar actuators that have only two polyphase armature conductors.

\section{Magnetically levitated MDOF planar actuator}

This chapter presents a feasibility verification as to whether a planar actuator can magnetically suspend a mover, capable of 3-DOF motions on a plane, so as to further improve the drive performance of a planar actuator.

\subsection{Actuator design}

The proposed planar actuator has spatially superimposed magnetic circuits for the $x-, y-$ and $\alpha$-directions, which are its most important feature and enable the mover to travel over a wide movable area on a plane by exciting only two polyphase armature conductors. The magnetically levitated planar actuator is also designed so that all the magnetic circuits are mutually superimposed. In order to design the planar actuator, a numerical analysis of 6DOF driving forces for 6-DOF mover positions is performed. 


\subsubsection{6-DOF force analysis}

The driving forces, including the suspension forces, greatly depend on the size of the gap between the mover and armature conductors, and therefore this gap needs to be precisely controlled. Generally, reducing this gap increases the driving forces. If the mover is located below the stator, attraction forces to the stator are required to suspend the mover. However, the attraction forces are increased by reducing the gap, which makes the vertical motions of the mover unstable. Conversely, if the mover is located above the stator, repulsion forces from the stator are required to suspend the mover. The repulsion forces are increased by reducing the gap, and so the vertical motions are stable. Therefore, in this study, the mover of the magnetically levitated planar actuator is positioned on the stator.

Figure 15 shows the analytical model for the driving forces. In this figure, the mover and polyphase armature conductors for the $x$-direction only are shown. A moving 2-D Halbach permanent-magnet array has the same structure as shown in Fig. 3, and four-pole-andseven-segment magnetization with pole-pitch length $\tau_{P M}=3 \mathrm{~mm}$ along the $x_{l}$ and $y_{l}$ directions. Its dimensions are $11 \mathrm{~mm} \times 11 \mathrm{~mm} \times 2 \mathrm{~mm}$, which are almost two-fifths the size of the magnet-array dimension shown in Fig. 3. The ultimate miniaturization of the permanent-magnet mover enables higher accelerations to be generated using the same armature currents and flux density as given in Subsection 2.2.1.

In the mover motions, there are 3-DOF rotations. However, this analysis deals with the rotations around only one axis $\left(x_{m}, y_{m}\right.$, or $\left.z_{m}\right)$. The rotational angles around the $x_{m-}, y_{m^{-}}$, and $z_{m}$-axes are referred to as roll angle $\gamma$, pitch angle $\beta$, and yaw angle $\alpha$, respectively. The driving forces acting on the mover can be calculated from the Lorentz force law with the same equations as Eqs. (4) and (5).

Figure 16 shows the analysis results of the driving forces $F_{x}, F_{z}, T_{x}, T_{y}, T_{z}$ for the yaw angle $\alpha$ when the $d$ - and $q$-axis currents for the $x$-directional drive are supplied $\left(I_{d x}=1 \mathrm{~A}\right.$, or $I_{q x}=1$ A), the air gap between the mover bottom and armature conductors is $0.5 \mathrm{~mm}$, and

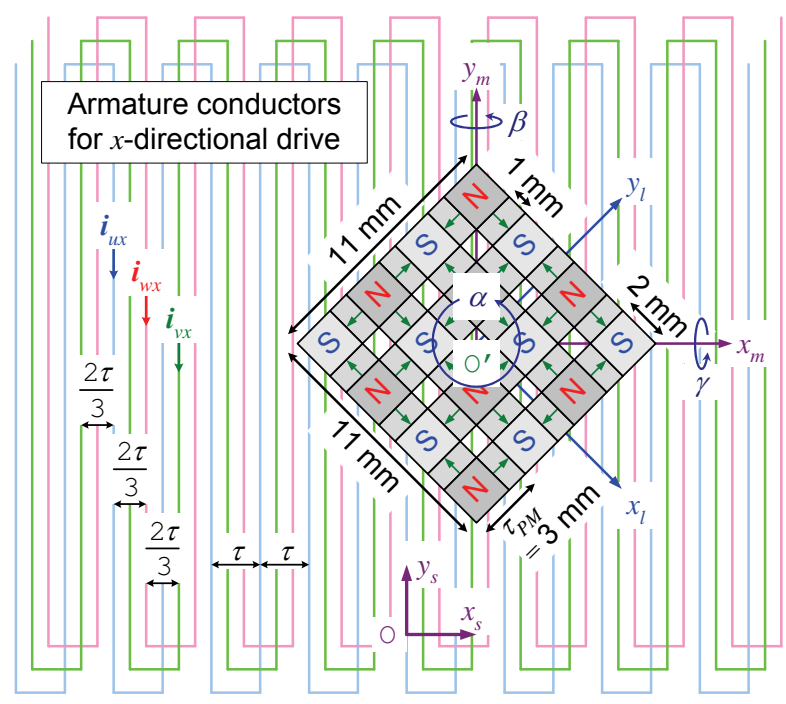

Fig. 15. Analytical model for 6-DOF driving forces 
the pitch and roll positions are not displaced $(\beta=\gamma=0 \mathrm{deg})$. Figure 16 indicates that the $d$-axis current generates the translational forces $F_{z}$ and torques $T_{z}$, and the $q$-axis current generates the translational forces $F_{x}$ and torques $T_{x}, T_{y}$. The translational forces $F_{x}, F_{z}$ and torques $T_{y}$ are almost constant, and the torques $T_{x}$ and $T_{z}$ are proportional to the yaw angle $\alpha$ when the yaw angle $\alpha \approx 0 \mathrm{deg}$. Because of the symmetric magnetization of the mover, the same driving forces can be generated every $180 \mathrm{deg}$.

From the analysis results shown above and others for the pitch angle $\beta$ and the roll angle $\gamma$, the driving forces $F_{x}, F_{y}, F_{z}, T_{x}, T_{y}, T_{z}$ can be expressed from the $d$ - and $q$-axis currents $I_{d x}, I_{q x}$, $I_{d y}, I_{q y}$ as follows:

$$
\left[\begin{array}{c}
F_{x} \\
F_{y} \\
F_{z} \\
T_{x} \\
T_{y} \\
T_{z}
\end{array}\right]=\left[\begin{array}{c}
\boldsymbol{K}_{F T}(\alpha, \beta \gamma) \\
6 \times 4 \text { matrix }
\end{array}\right]\left[\begin{array}{c}
I_{d x} \\
I_{q x} \\
I_{d y} \\
I_{q y}
\end{array}\right] \approx\left[\begin{array}{cccc}
0 & K_{F} & 0 & 0 \\
0 & 0 & 0 & K_{F} \\
-K_{F} & 0 & -K_{F} & 0 \\
K_{T P} \gamma & K_{T P} \alpha & K_{T P} \gamma & K_{T C} \\
K_{T P} \beta & -K_{T C} & K_{T P} \beta & K_{T P} \alpha \\
-K_{T P} \alpha & K_{T P} \gamma & -K_{T P} \alpha & K_{T P} \beta
\end{array}\right]\left[\begin{array}{c}
I_{d x} \\
I_{q x} \\
I_{d y} \\
I_{q y}
\end{array}\right]
$$

where $K_{F T}$ is a $6 \times 4$ matrix and all elements of the matrix nonlinearly depend on the yaw angle $\alpha$, pitch angle $\beta$, and roll angle $\gamma$. In this study, the pitch and roll displacements of the mover are assumed to be very small ( $\beta \approx 0 \mathrm{deg}$ and $\gamma \approx 0 \mathrm{deg}$ ) because of small air gap (less than $1 \mathrm{~mm}$ ) between the mover and stator, and in the range, all elements of $\boldsymbol{K}_{F T}$ almost linearly depend on the pitch and roll displacements. Furthermore, if the yaw displacements are assumed also to be very small $(\alpha \approx 0 \mathrm{deg})$, all elements of $K_{F T}$ almost linearly depend on the yaw displacements, and the system-constant matrix $K_{F T}$ is expressed approximately as shown in Eq. (20).

In Eq. (20), $K_{F C}, K_{T C}$, and $K_{T P}$ are constant (in this analysis, for a $0.5-\mathrm{mm}$ air gap, $K_{F C} \approx 17 \mathrm{mN}$, $K_{T C} \approx 12 \mathrm{mN} \cdot \mathrm{mm}$, and $K_{T P} \approx 4.5 \mathrm{mN} \cdot \mathrm{mm}$ ). Equation (20) also indicates that the driving forces due to the $d$-axis currents $I_{d x}$ and $I_{d y}$ are equal because of the symmetry of the actuator.

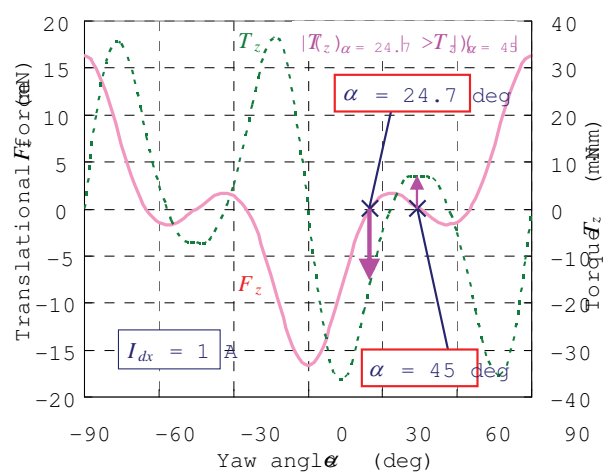

(a) Driving forces from $I_{d x}=1 \mathrm{~A}$.

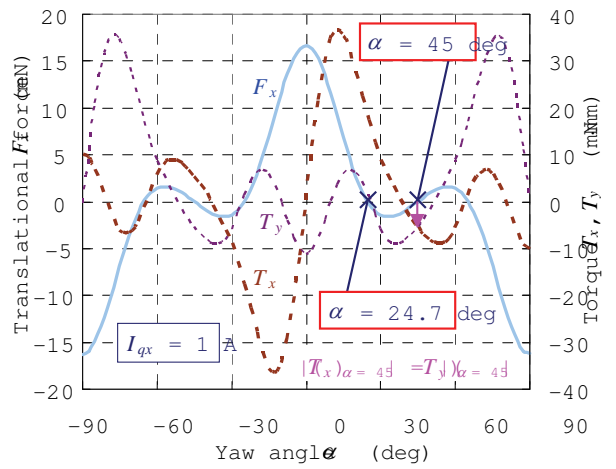

(b) Driving forces from $I_{q x}=1 \mathrm{~A}$.

Fig. 16. Driving forces for yaw angle $\alpha$ at pitch and roll angles $\beta=\gamma=0 \mathrm{deg}$ 
Therefore, even if the two currents $I_{d x}$ and $I_{d y}$ are controlled, only 1-DOF driving forces can be controlled in the range within $\alpha \approx 0 \mathrm{deg}, \beta \approx 0 \mathrm{deg}$, and $\gamma \approx 0 \mathrm{deg}$. Therefore, controlling the four armature currents in the $d q$-frame controls the 3-DOF motions of the mover (for instance, $x-, y-$ and $z$-motions, or $x-, y-$, and $\alpha$-motions). In order to realize both 3-DOF motion controls on a plane and magnetic suspension, the planar actuator needs to be redesigned.

\subsubsection{Conceptual design}

In order to suspend the mover, suspension forces that balance the force of gravity need to be generated. Equation (20) indicates that negative $d$-axis currents $\left(I_{d x}, I_{d y}<0\right)$ generate suspension forces $\left(F_{z}>0\right)$. Figure 17 shows schematic views of when the $d$-axis currents are supplied. Negative $d$-axis currents to actively control levitation forces $\left(F_{z}>0\right)$ always generate restoring torques against the $\beta$ - and $\gamma$-displacements. The restoring torques stabilize the $\beta$ - and $\gamma$-motions of the mover.

Equation (20) also shows that the $q$-axis currents $I_{q x}, I_{q y}$ generate the translational forces $F_{x}$, $F_{y}$ on a plane without vertical forces $F_{z}$. Therefore, the $d$ - and $q$ - axis currents $I_{d x}, I_{q x}, I_{d y}, I_{q y}$ :

- $\quad$ independently control the translational forces $F_{x}, F_{y}, F_{z}$

- $\quad$ stabilize the pitch and roll motions.

However, the $d$-axis currents utilized to control the suspension forces $F_{z}$, generate yawdirectional torques proportional to the yaw angle $\alpha$, that is, they generate instable yaw motions. Therefore, in order to realize both 3-DOF motion controls on a plane and magnetic suspension, a stabilization mechanism for the yaw motions is needed.

The torques acting on the mover depend on the relative yaw, pitch, and roll distances between the mover and the armature conductors, but relative pitch and roll distances should be always nearly equal to 0 deg in order to maintain a small air gap. In this study, new armature conductors with different relative distances in the yaw direction from the armature conductors for the $x$ - and $y$-directional drives are introduced to control the yaw motion.

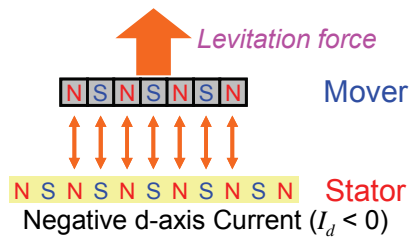

(a) Levitation forces $F_{z}$.

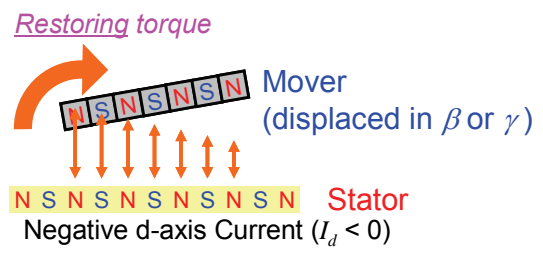

(b) Restoring torques $T_{y}$ and $T_{x}$.

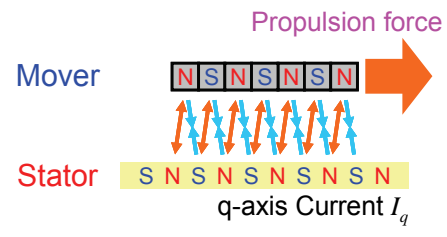

(c) Propulsion forces $F_{x}$ and $F_{y}$.

Fig. 17. Conceptual design of a magnetically levitated planar actuator 
Figure 16 indicates that the $d$-axis current generates translational forces $F_{z}$ and torques $T_{z}$, and the $q$-axis current generates translational forces $F_{x}, F_{y}$ and torques $T_{x}, T_{y}$ when the pitch and roll positions are not displaced $(\beta=\gamma=0 \mathrm{deg})$. So, at least four kinds of the $q$-axis currents, that is, four pairs of polyphase currents are needed to actively control 6-DOF motions. Furthermore, Fig. 16 also indicates that the $d$ - and $q$-axis currents generate only torques without translational forces when the relative yaw distance is $24.7 \mathrm{deg}$ or $45 \mathrm{deg}$. As mentioned in Subsection 3.1.1, a magnitude of torque $T_{z}$ resulting from the mover tiled by $24.7 \mathrm{deg}$ is larger than that by $45 \mathrm{deg}$. Therefore in this study, the armature conductors are tilted by $24.7 \mathrm{deg}$ in the yaw direction from the armature conductors for the $x$-directional drive, I term this arrangement "armature conductors for the $\alpha$-directional drive." When the yaw angle of the mover $\alpha=0 \mathrm{deg}$, the $d$-axis currents for the $\alpha$-directional drive $I_{d_{\alpha}}$ :

- $\quad$ generate only torques $T_{z}$

- $\quad$ without vertical forces $F_{z}$.

Therefore, the $d$-axis currents $I_{d_{\alpha}}$ can separate the generation of the vertical forces $F_{z}$ and torques $T_{z}$, and stabilize the yaw motion. To date, the $d$ - and $q$-axis currents are generated by three-phase currents, but they can be also be generated by two-phase currents. In this study, a magnetically levitated planar actuator with three pairs of two-phase armature conductors is organized as shown in Fig. 18.

\subsection{Dynamic behavior of mover}

The mover has 3-DOF translational and rotational motions because there is no mechanical suspension mechanism. When the physical quantities of the mover motion are represented,

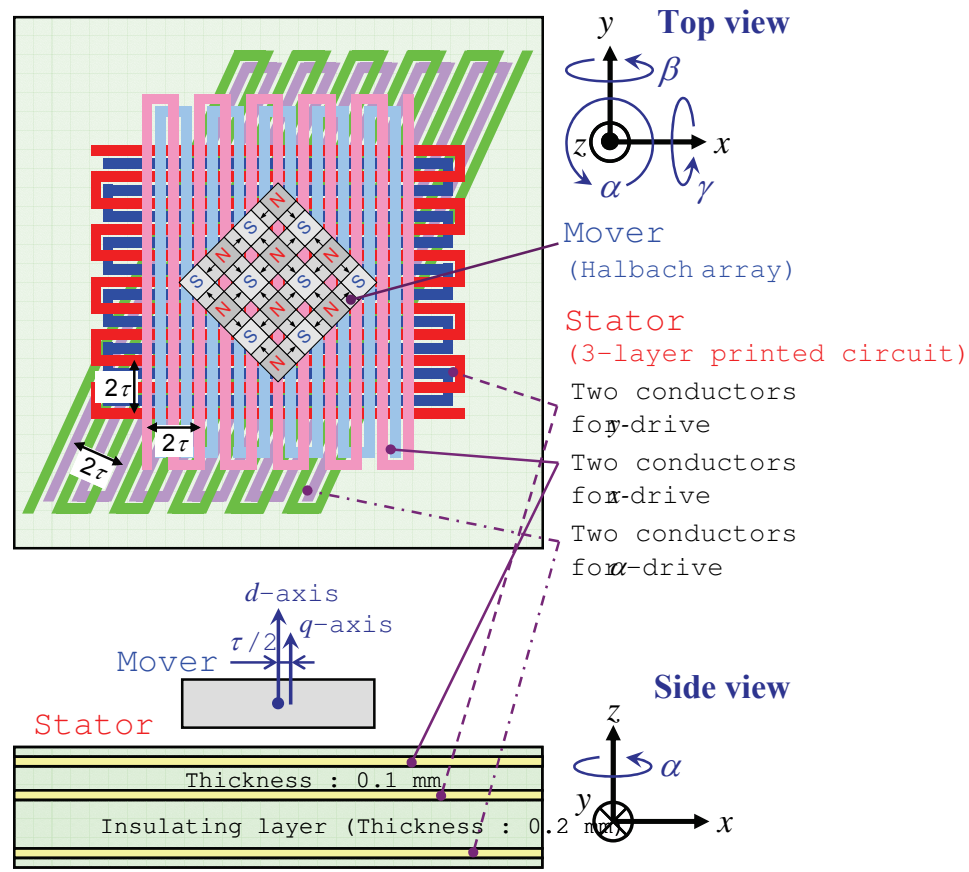

Fig. 18. Fundamental structure of magnetically levitated planar actuator 
it is extremely important what coordinates are respected. The translational motions are often represented with respect to the stationary coordinate, and the rotational motions are often represented with respect to the mover coordinate. This section introduces an equation for the 6-DOF motions of the mover that describes the dynamic behavior.

The equation of the motion of the mover can be represented by the translational forces acting on the mover $\boldsymbol{F}_{s m}=\left[\begin{array}{llll}F_{x} & F_{y} & F_{z}\end{array}\right]^{T}$ and torques around the mover center $\mathrm{O}^{\prime} \boldsymbol{T}_{s m}{ }^{\prime}=\left[\begin{array}{lll}T_{x}{ }^{\prime} & T_{y}{ }^{\prime}\end{array}\right.$ $\left.T_{z}{ }^{\prime}\right]^{T}$ from mass $M$ and inertia tensor $\boldsymbol{J}_{m}{ }^{\prime}$ of the mover as follows:

$$
\begin{gathered}
M \frac{d \boldsymbol{v}_{s m}}{d t}=\boldsymbol{F}_{s m}+\boldsymbol{F}_{g} \\
\boldsymbol{J}_{m}{ }^{\prime} \frac{d \boldsymbol{\omega}_{s m}{ }^{\prime}}{d t}=\boldsymbol{T}_{s m} \stackrel{+}{\boldsymbol{\omega}_{s m}} \times\left(\begin{array}{ll}
\boldsymbol{J}_{m} & \boldsymbol{\omega}_{s m}^{\prime}
\end{array}\right),
\end{gathered}
$$

where $\boldsymbol{v}_{s m}=\left[\begin{array}{lll}v_{x} & v_{y} & v_{z}\end{array}\right]^{T}$ and $\boldsymbol{F}_{g}=\left[\begin{array}{lll}0 & 0 & -M g\end{array}\right]^{T}$ are velocity of the mover and the force of gravity acting on the mover, respectively.

Equations (21) and (22) represent 3-DOF translational and rotational motion equations of the mover, respectively. All variables in the translational and rotational motion equations are represented with respect to the stationary coordinate $x_{s} y_{s} z_{s}$ and mover coordinate $x_{m} y_{m} z_{m}$, respectively. The position $\boldsymbol{r}_{s m}=\left[\begin{array}{lll}x & y & z\end{array}\right]^{T}$, with respect to the stationary coordinate $x_{s} y_{s} z_{s}$, and Euler angle $\phi=\left[\begin{array}{ccc}\alpha & \beta & \gamma\end{array}\right]^{T}$, which is defined from $\alpha, \beta$, and $\gamma$ as orderly counterclockwise rotations about the $z-, y$ - and $x$-axes, respectively, can be represented by the velocity $v_{s m}$ and angular velocity $\omega_{s m^{\prime}}$, respectively, as follows:

$$
\begin{gathered}
\frac{d \boldsymbol{r}_{s m}}{d t}=\boldsymbol{v}_{s m} \\
\frac{d \boldsymbol{\varphi}}{d t}=\boldsymbol{R}_{\omega \varphi}(\boldsymbol{\varphi})^{-1} \boldsymbol{\omega}_{s m},
\end{gathered}
$$

where $\boldsymbol{R}_{\omega \phi}$ is a $3 \times 3$ matrix and all elements of the matrix nonlinearly depend on the Euler angle $\phi$. Equations (21)-(24) can represent dynamic behaviors of the mover with 6 DOF.

\subsection{Planar motion control with stable magnetic levitation}

This section discusses six-current controls to stably levitate the mover and actively control the $x-, y-, z-$, and $\alpha$-motions. There are two important things for the motion controls:

- to generate independent translational forces $F_{x}, F_{y}$, and $F_{z}$ with stable torques in the $\gamma$ and $\beta$-directions.

- to generate torques in the $\alpha$-direction with less interference to translational forces $F_{x}, F_{y}$, and $F_{z}$.

This section first presents driving forces resulting from three pairs of two-phase armature currents, and then the driving force-control system.

\subsubsection{Translational motion control}

In this study, three pairs of two-phase currents $i_{j}=\left[\begin{array}{ll}I_{1 j} & I_{2 j}\end{array}\right]^{T}(j=x, y$, or $\alpha)$ are assumed to be supplied to the three pairs of two-phase armature conductors as shown in the following equations: 


$$
\begin{gathered}
\left.I_{1 j}=-I_{j} \operatorname{co} \oint \theta_{s j}\right) \\
I_{2 j}=I_{j} \operatorname{sif}\left(\theta_{s j}\right)
\end{gathered}
$$

Figure 19 shows phasor diagrams for the relation between the $d q$-frame and $\alpha^{\prime} \beta^{\prime}$-frame. The currents $I_{1 x}$ and $I_{1 y}$ generate the opposite-phase magnetic field to that resulting from the permanent-magnet mover when the mover position in the $x$ - and $y$-directions $(x, y)=\left(x_{s}, y_{s}\right)$ and the Euler angle $\phi=(0,0,0)$. The $\alpha^{\prime}$-axis are aligned to the opposite side of the current $I_{1 j}$ axis, and the $\beta^{\prime}$-axis leads the $\alpha^{\prime}$-axis by $90 \mathrm{deg}$. The current $I_{1 \alpha}$ generates a magnetic field that is tilted by $\varphi=-24.7 \mathrm{deg}$ around the $\alpha$-direction from that caused by current $I_{1 x}$. Bearing this in mind, the armature currents in the $d q$-frame $I_{d j}$ and $I_{q j}$ can be represented by the currents $I_{1 j}$ and $I_{2 j}$ as follows $(j=x, y$, or $\alpha)$ :

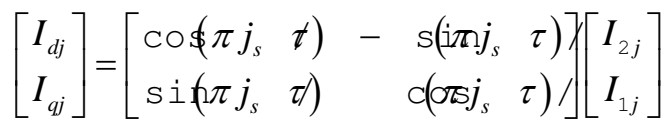

$$
\begin{aligned}
& \alpha_{s}=x_{s} \cos \phi-y_{s} \quad \mathrm{si \phi n}
\end{aligned}
$$

These pairs of $d$ - and $q$-axis currents generate the translational forces $\boldsymbol{F}_{s m}$ and torques $\boldsymbol{T}_{s m}{ }^{\prime}$ as follows:

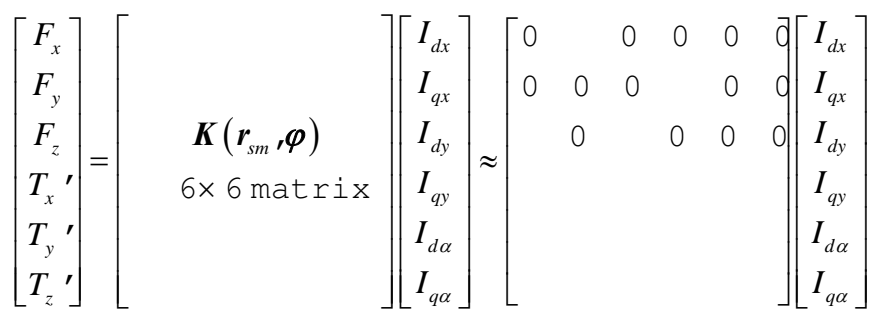

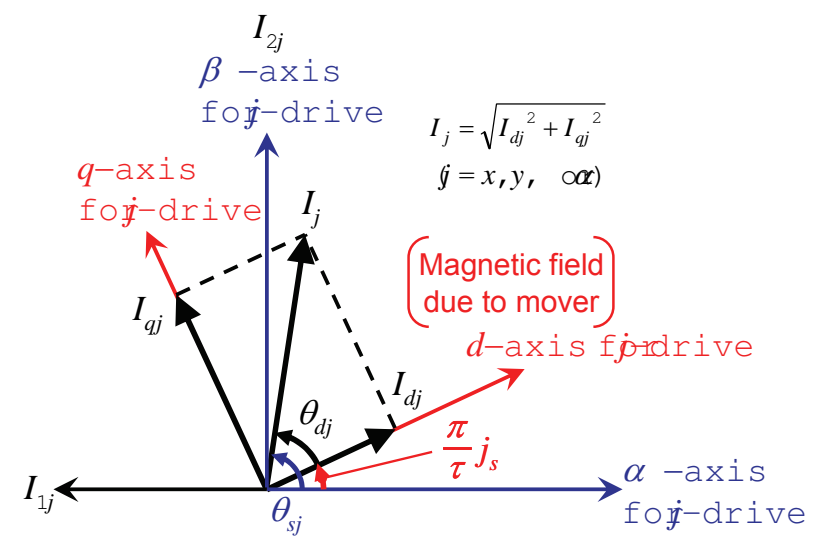

Fig. 19. Phasor diagram showing relation between $d q$-frame and $\alpha^{\prime} \beta^{\prime}$-frame 
where $K$ is a $6 \times 6$ matrix, and all elements of $K$ depend on the mover position $\boldsymbol{r}_{s m}$ and Euler angle $\boldsymbol{\phi}$. Where Euler angle $\boldsymbol{\phi} \approx \mathbf{0}, K$ can be approximated as shown in Eq. (29), and therefore 3-DOF translational forces $F_{x}, F_{y}$, and $F_{z}$ can be independently controlled by two-phase currents $\boldsymbol{i}_{x}$ and $\boldsymbol{i}_{y}$.

In this study, references of the translational forces $\boldsymbol{F}_{s m}{ }^{*}=\left[\begin{array}{lll}F_{x}^{*} & F_{y}{ }^{*} & F_{z}{ }^{*}\end{array}\right]^{T}$ are determined from the mover positions $\boldsymbol{r}_{s m}=\left[\begin{array}{lll}x & y & z\end{array}\right]^{T}$ and position references $\boldsymbol{r}_{s m}{ }^{*}=\left[\begin{array}{lll}x^{*} & y^{*} & z^{*}\end{array}\right]^{T}$ by three PID controls.

$$
\boldsymbol{F}_{s m}{ }^{\star}=\boldsymbol{P}_{F}\left(\boldsymbol{r}_{s m}{ }^{\star}-\boldsymbol{r}_{s m}\right)-\boldsymbol{D}_{F} \frac{d \boldsymbol{r}_{s m}}{d t}-\boldsymbol{F}_{g}
$$

where $\boldsymbol{P}_{F}=\operatorname{diag}\left(P_{F x}, P_{F y}, P_{F z}\right)$ and $\boldsymbol{D}_{F}=\operatorname{diag}\left(D_{F x}, D_{F y}, D_{F z}\right)$ are proportional and differential parameters, respectively. In this study, references of the armature currents $\boldsymbol{i}_{x}{ }^{*}$ and $\boldsymbol{i}_{y}{ }^{*}$ are calculated from those of the translational forces $\boldsymbol{F}_{s m}{ }^{*}$ as follows:

$$
\begin{aligned}
& {\left[\begin{array}{c}
I_{d x}^{*} \\
I_{q x}^{*}
\end{array}\right]=\left[\begin{array}{ll}
K_{11} & K_{12} \\
K_{31} & K_{32}
\end{array}\right]^{-1}\left[\begin{array}{c}
F_{x}^{*} \\
F_{z}^{*} / 2
\end{array}\right]} \\
& {\left[\begin{array}{l}
I_{d y}^{*} \\
I_{q y}^{*}
\end{array}\right]=\left[\begin{array}{ll}
K_{23} & K_{24} \\
K_{33} & K_{34}
\end{array}\right]^{-1}\left[\begin{array}{c}
F_{y}^{*} \\
F_{z}^{*} / 2
\end{array}\right]}
\end{aligned}
$$

Supplying the armature currents $\boldsymbol{i}_{x}$ and $\boldsymbol{i}_{y}$ equal to the references $\boldsymbol{i}_{x}{ }^{*}$ and $\boldsymbol{i}_{y}{ }^{*}$ generates the translational forces $\boldsymbol{F}_{s m}$ equal to the references $\boldsymbol{F}_{s m}{ }^{*}$.

\subsubsection{Rotational motion control}

The armature currents $\boldsymbol{i}_{x}$ and $\boldsymbol{i}_{y}$ generate not only the translational forces $\boldsymbol{F}_{\mathrm{sm}}$, but also the torques $\boldsymbol{T}_{s m}{ }^{\prime}$. Therefore, it is extremely important to investigate how the torques $\boldsymbol{T}_{s m}{ }^{\prime}$ resulting from the armature currents $i_{x}$ and $i_{y}$ influence the rotational motions of the mover. When the Euler angle $\phi \approx 0$, the torques $T_{z}{ }^{\prime}, T_{y}{ }^{\prime}$, and $T_{x}{ }^{\prime}$ are dominant on the Euler angle $\alpha, \beta$, and $\gamma$, respectively. Next I performed a numerical analysis of the torque characteristics due to the armature currents for the $x$-directional drive when rotational motions with more than 1 DOF occur.

Furthermore, from analysis results of the 6-DOF driving forces, when rotational motions with more than 1 DOF occur in the range within $-2 \mathrm{deg}<\alpha, \beta$, and $\gamma<2 \mathrm{deg}$, a $6 \times 4$ submatrix of $\boldsymbol{K}$ is almost in agreement with $K_{F T}$ in Eq. (20). Therefore, negative $d$-axis currents $I_{d x}, I_{d y}$ that control the suspension forces $F_{z}$ generate stable restoring torques $T_{y}{ }^{\prime}, T_{x}{ }^{\prime}$. However, the $q$-axis currents that control the translational forces $F_{x}, F_{y}$ generate torques $T_{z}{ }^{\prime}$, $T_{y}{ }^{\prime}, T_{x}{ }^{\prime}$, which are not stable restoring torques. So next I performed a numerical analysis of the torque characteristics due to the armature currents for the $\alpha$-directional drive.

Figure 20 shows the torques due to the armature conductors for the $\alpha$-directional drive at $(\beta, \gamma)=(0,0)$. When the Euler angles $(\beta, \gamma)=(0,0)$, the $d$-axis current $I_{d \alpha}$ generates only the torque $T_{z}{ }^{\prime}$ and the $q$-axis current $I_{q \alpha}$ generates only the torques $T_{y}{ }^{\prime}, T_{x}{ }^{\prime}$. Therefore, the torques $T_{y}{ }^{\prime}$ and $T_{x}{ }^{\prime}$ cannot be independently controlled by the armature currents for the $\alpha$-directional drive. When the Euler angle $\phi \approx 0$ and angular velocity $\omega_{m s}{ }^{\prime} \approx 0$, a linearized equation of the rotational motion can be obtained from Eqs. (22) and (24) as follows: 


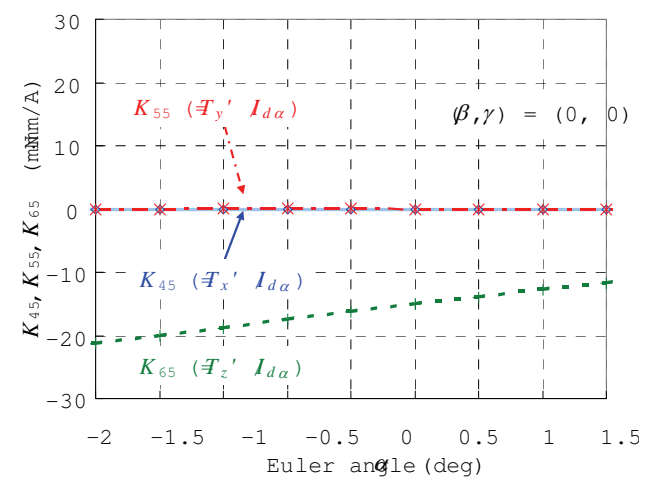

(a) $K_{45}, K_{55}$, and $K_{65}$.

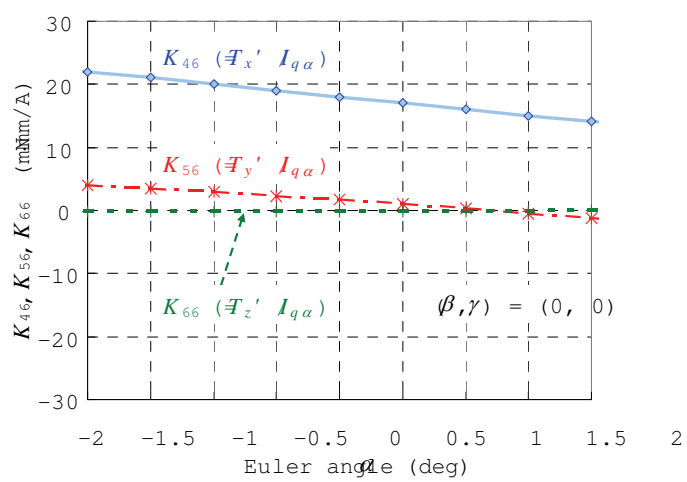

(b) $K_{46}, K_{56}$, and $K_{66}$.

Fig. 20. Analysis result of the torques from the armature conductors for the $\alpha$-drive

$$
\frac{d^{2} \boldsymbol{\varphi}}{d t^{2}} \approx \boldsymbol{R}_{\omega \varphi}\left(\boldsymbol{J}_{m}\right)^{\prime-1} \boldsymbol{T}_{s m} \pm \boldsymbol{T}_{E}=\left[\begin{array}{lll}
T_{\alpha} & T_{\beta} & T_{\gamma}
\end{array}\right]^{T} .
$$

In this study, $\boldsymbol{T}_{E}{ }^{*}$, which is the reference of $\boldsymbol{T}_{E}$, is determined by a PD control from the Euler angle $\alpha$ and the reference $\alpha^{*}$ as follows:

$$
T_{\alpha}^{*}=P_{T \alpha}\left(\alpha^{\star}-\alpha\right)-D_{T \alpha} \frac{d \alpha}{d t}
$$

where $P_{T_{\alpha}}$ and $D_{T_{\alpha}}$ are proportional and differential parameters, respectively. Then, the references $T_{\beta}{ }^{*}$ and $T_{\gamma}^{*}$ are determined to be zero because of the suppression of the $\beta$ - and $\gamma$-motions. The torque references $T_{x}{ }^{\prime *}$ and $T_{z}{ }^{\prime *}$ can be calculated from the reference $\boldsymbol{T}_{E}{ }^{*}$ by Eq. (33). Then, the references of the armature currents for the $\alpha$-directional drive $I_{d \alpha}{ }^{*}$ and $I_{q \alpha}{ }^{*}$ can be calculated for the torque references $T_{x}{ }^{\prime *}$ and $T_{z}{ }^{\prime *}$ as follows:

$$
\left[\begin{array}{c}
I_{d \alpha}{ }^{*} \\
I_{q \alpha}{ }^{*}
\end{array}\right]=\left[\begin{array}{ll}
K_{45} & K_{46} \\
K_{65} & K_{66}
\end{array}\right]^{-1}\left(\left[\begin{array}{c}
T_{x}{ }^{\dagger} \\
T_{z}^{\prime}
\end{array}\right]-\left[\begin{array}{l}
T_{x a}^{\prime} \\
T_{z a}^{\prime}
\end{array}\right]\right)
$$

where $T_{x a}{ }^{\prime}$ and $T_{z a}{ }^{\prime}$ are torques due to the armature currents $\boldsymbol{i}_{x}$ and $\boldsymbol{i}_{y}$, and can be represented as follows:

$$
\left[\begin{array}{c}
T_{x a} \\
T_{z a}
\end{array}\right]=\left[\begin{array}{llll}
K_{41} & K_{42} & K_{43} & K_{44} \\
K_{61} & K_{62} & K_{63} & K_{64}
\end{array}\right]\left[\begin{array}{c}
I_{d x} \\
I_{q x} \\
I_{d y} \\
I_{q y}
\end{array}\right] .
$$

Supplying the armature currents $\boldsymbol{i}_{\alpha}$ equal to the references $\boldsymbol{i}_{\alpha}{ }^{*}$ generates $\boldsymbol{T}_{E}$ nearly equal to $\boldsymbol{T}_{E}{ }^{*}$, and controls the rotational motions with less interference to the translational motions. 


\subsection{Numerical analysis of mover motion}

Motion characteristics with 6 DOF can be obtained by solving Eqs. (21)-(24) using the Runge-Kutta method. In order to numerically solve the equations, it is necessary to calculate the driving forces $\boldsymbol{F}_{s m}$ and $\boldsymbol{T}_{s m}{ }^{\prime}$ at each time step. The calculation at each time step consists of an integration of Lorentz force acting on the line segments as shown in Eqs. (4) and (5), and so requires a lot of computation time. The flux density $\boldsymbol{B}$ acting on the armature conductors greatly depends on the mover position $\boldsymbol{r}_{s m}$ and Euler angle $\phi$. Therefore, the driving forces $\boldsymbol{F}_{s m}$ and $\boldsymbol{T}_{s m}$ ' are functions of the mover position $\boldsymbol{r}_{s m}$ and Euler angle $\boldsymbol{\phi}$. In this study, the system-constant matrix $\boldsymbol{K}$ was calculated and the data table of $\boldsymbol{K}$ was made before the motion analysis. Then, the system-constant matrix $K$ is calculated from the mover position $\boldsymbol{r}_{s m}$ and Euler angle $\phi$ by interpolating it with the data table at each time step. The analysis conditions are shown as follows:

- $\quad$ time step $d t=0.2 \mathrm{~ms}$

- control period $t_{c}=2 \mathrm{~ms}$

- $\quad$ initial position $\boldsymbol{r}_{i}=\mathbf{0}$

- $\quad$ initial Euler angle $\boldsymbol{\phi}_{i}=\mathbf{0}$.

When the $z$-position is zero, the mover is assumed to be on the stator. The proportional and differential parameters are determined so that the settling times in the $x_{-}, y_{-}, z_{-}$, and $\alpha$-motions are less than $1 \mathrm{~s}$. In this analysis, to investigate the planar motion control and magnetic levitation, the following position reference is given:

- $x^{*}=2 \cos (\pi t) \mathrm{mm}$

- $y^{*}=2 \sin (\pi t) \mathrm{mm}$

- $z^{*}=0.15 \mathrm{~mm}$

- $\quad$ Euler angle $\alpha^{*}=0$ deg.

Figure 21 shows the analysis result of the mover motions under this analysis condition, and indicates that the mover can track the reference positions in the $x$ - and $y$-directions, and be positioned in the $z$ - and $\alpha$-directions with suppression of the $\beta$ - and $\gamma$-displacements. Therefore, mover motions can be controlled with stable magnetic levitation. The $q$-axis currents $I_{q x}$ and $I_{q y}$ used to control the translational forces $F_{x}$ and $F_{y}$ also generate simultaneously the torques $T_{y}{ }^{\prime}$ and $T_{x}{ }^{\prime}$, respectively. Therefore, displacement of the Euler angles $\beta$ and $\gamma$ slightly occurs.

\subsection{Summary of chapter 3}

This chapter presents a feasibility verification of a planar actuator with both 3-DOF planar motions and magnetic suspension of the mover in order to further improve performance. Then, based on a numerical analysis of the 6-DOF driving forces, a planar actuator having a mover positioned above a plane and magnetically levitated by only six currents and the sixcurrent-control algorithm were conceptually designed. Furthermore, I validated the designed planar actuator by numerical analysis of the 6-DOF motions. The results obtained in this paper indicate the possibility of the realization of a high-performance MDOF planar actuator:

- decoupled 3-DOF motion control and magnetic levitation on a plane.

- $\quad$ wide movable area by a small number (six) of armature conductors.

- $\quad$ extendible movable area regardless of the number of armature conductors.

- $\quad$ small millimeter-sized mover.

- $\quad$ no problematic wiring to adversely affect drive performance. 


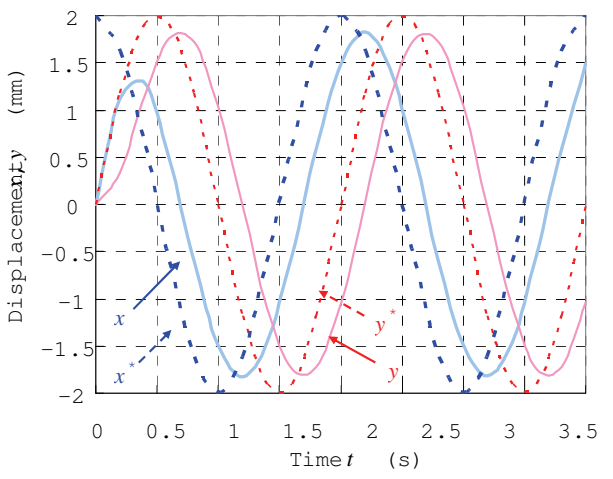

(a) Planar motions $x, y$.

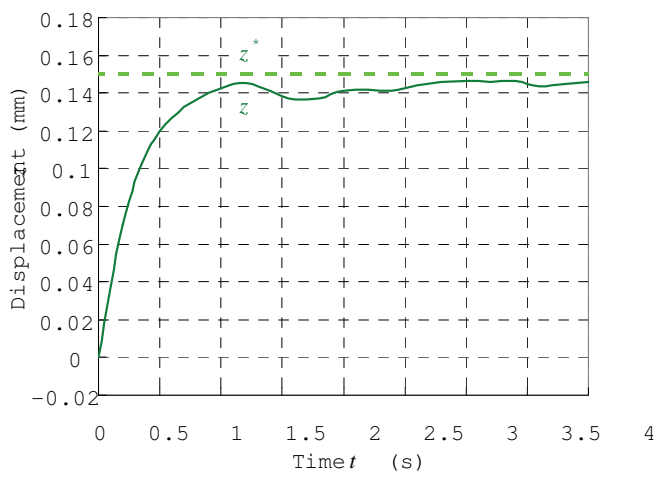

(b) Vertical motion $z$.

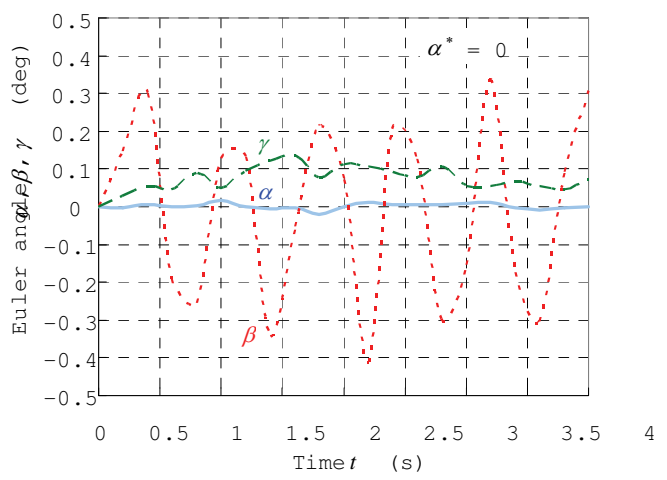

(c) Rotational motions $\alpha, \beta, \gamma$.

Fig. 21. Analytically-obtained 6-DOF mover motions (Ueda \& Ohsaki, 2008b)

\section{Conclusion}

This paper presents high-performance MDOF planar actuators with a permanent-magnet mover capable of traveling over a wide movable area on a plane, with just a small number of stationary armature conductors. The combination of the mover and stator can generate spatially superimposed magnetic fields for the MDOF drive, and therefore increasing the length of the armature conductors can easily expand the movable area regardless of the number of armature conductors. A planar actuator was conceptually designed and fabricated. The fabricated planar actuator can independently control the 3-DOF motions of the mover. Furthermore, in order to eliminate deterioration of the drive characteristics due to friction forces, the planar actuator was redesigned so that the mover could be stably levitated and the 3-DOF motions on a plane could be controlled. Then, the mover motion characteristics were successfully verified by means of a numerical analysis. Next, a small fabrication size was realized by integrating the permanent-magnet array and armature conductors for the MDOF drive. The planar actuator has the first millimeter-sized mover and would provide a significant starting point when used with small electromechanical components in an MDOF drive. 


\section{References}

Ebihara, D.; Wakui, S.; Tsuchiya, J.; Inui, S.; Wakiwaka, H.; Ohsaki, H.; Yano, T.; Maeno, T.; Watanabe, T.; Hirata, K.; Dohmeki, H.; Ohashi, K.; Tomita, Y.; Maemura, A \& Takemura, K. (2005). Investigation of Possibility of Multi-Dimensional Drive System, The Institute of Electrical Engineers of Japan, ISSN 0919-9195, MARUI-Kobunsha Corp.

Fitzgerald, A. E.; Kingsley, C. Jr. \& Umans, S. D. (1990). Electric Machinery, McGraw Hill Higher Education, ISBN 978-0073660097

Halbach, K. (1986). Concepts for Insertion Devices that will Produce High-Quality Synchrotron Radiation. Nuclear Instruments and Methods in Physics Research Section A, Vol.246, No.1-3, May 1986, pp.77-81, ISSN 0168-9002

Jeon, J. W.; Caraiani, M.; Kim, Y. J.; Oh, H. S. \& Kim, S. S. (2007). Development of Magnetic Levitated Stage for Wide Area Movements. Proceedings of the International Conference on Electrical Machines and Systems, pp.1486-1491, Korea, October 2007, Seoul

Kim, W. J. \& Trumper, D. 


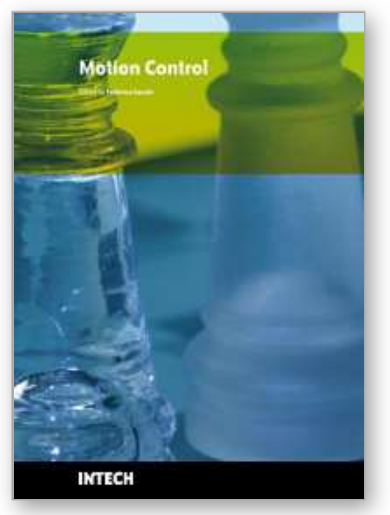

\author{
Motion Control \\ Edited by Federico Casolo
}

ISBN 978-953-7619-55-8

Hard cover, 590 pages

Publisher InTech

Published online 01, January, 2010

Published in print edition January, 2010

The book reveals many different aspects of motion control and a wide multiplicity of approaches to the problem as well. Despite the number of examples, however, this volume is not meant to be exhaustive: it intends to offer some original insights for all researchers who will hopefully make their experience available for a forthcoming publication on the subject.

\title{
How to reference
}

In order to correctly reference this scholarly work, feel free to copy and paste the following:

Yasuhito Ueda and Hiroyuki Ohsaki (2010). A Long-Stroke Planar Actuator with Multiple Degrees of Freedom by Minimum Number of Polyphase Currents, Motion Control, Federico Casolo (Ed.), ISBN: 978-953-7619-558, InTech, Available from: http://www.intechopen.com/books/motion-control/a-long-stroke-planar-actuator-withmultiple-degrees-of-freedom-by-minimum-number-of-polyphase-curren

\section{INTECH}

open science | open minds

\author{
InTech Europe \\ University Campus STeP Ri \\ Slavka Krautzeka 83/A \\ 51000 Rijeka, Croatia \\ Phone: +385 (51) 770447 \\ Fax: +385 (51) 686166 \\ www.intechopen.com
}

\author{
InTech China \\ Unit 405, Office Block, Hotel Equatorial Shanghai \\ No.65, Yan An Road (West), Shanghai, 200040, China \\ 中国上海市延安西路65号上海国际贵都大饭店办公楼405单元 \\ Phone: +86-21-62489820 \\ Fax: $+86-21-62489821$
}


(C) 2010 The Author(s). Licensee IntechOpen. This chapter is distributed under the terms of the Creative Commons Attribution-NonCommercialShareAlike-3.0 License, which permits use, distribution and reproduction for non-commercial purposes, provided the original is properly cited and derivative works building on this content are distributed under the same license. 